FERMILAB-TM-2040

\title{
NuTeV SSQT Performance
}

\author{
J. Yu et al. \\ The NuTeV Collaboration \\ Fermi National Accelerator Laboratory \\ P.O. Box 500, Batavia, Illinois 60510
}

February 1998 


\section{Disclaimer}

This report was prepared as an account of work sponsored by an agency of the United States Government. Neither the United States Government nor any agency thereof, nor any of their employees, makes any warranty, expressed or implied, or assumes any legal liability or responsibility for the accuracy, completeness, or usefulness of any information, apparatus, product, or process disclosed, or represents that its use would not infringe privately owned rights. Reference herein to any specific commercial product, process, or service by trade name, trademark, manufacturer, or otherwise, does not necessarily constitute or imply its endorsement, recommendation, or favoring by the United States Government or any agency thereof. The views and opinions of authors expressed herein do not necessarily state or reflect those of the United States Government or any agency thereof.

\section{Distribution}

Approved for public release; further dissemination unlimited. 
FERMILAB-TM-2040

\title{
NuTeV SSQT Performance
}

\author{
J.Yu, ${ }^{3}$ K.S.McFarland, ${ }^{3}$ R.B.Drucker,${ }^{6}$ R.H.Bernstein, ${ }^{3}$ \\ P. Spentzouris, ${ }^{2}$ T. Bolton, ${ }^{4}$ J. Conrad, ${ }^{2}$ D. A. Harris,${ }^{7}$ \\ R. A. Johnson, ${ }^{1}$ G. Koizumi, ${ }^{3}$ M. J. Lamm, ${ }^{3}$ W. Marsh, ${ }^{3}$ \\ H. Schellman ${ }^{5}$ M. H. Shaevitz, ${ }^{2}$ M. Vakili, ${ }^{1}$ E. Villegas ${ }^{3}$ \\ ${ }^{1}$ University of Cincinnati, Cincinnati, OH 45221 \\ 2 Columbia University, New York, NY 10027 \\ ${ }^{3}$ Fermi National Accelerator Laboratory, Batavia, IL 60510 \\ ${ }^{4}$ Kansas State University, Manhattan, KS 66506 \\ ${ }^{5}$ Northwestern University, Evanston, IL 60208 \\ ${ }^{6}$ University of Oregon, Eugene, OR 97403 \\ ${ }^{7}$ University of Rochester, Rochester, NY 14627

\section{NuTeV Collaboration}

\begin{abstract}
The NuTeV experiment (E815) took data during the 1996-1997 Fermilab fixed target run. The experiment used the sign-selected-quadrupole-train (SSQT) in its neutrino beamline to choose either a neutrino or an antineutrino beam. This note summarizes the performance of the SSQT, as measured by the beamline monitoring devices, the observed $\nu_{\mu}$ and $\bar{\nu}_{\mu}$ fluxes, and the upper limit on the wrong sign neutrino contamination.
\end{abstract}




\section{Contents}

1 Introduction $\quad 3$

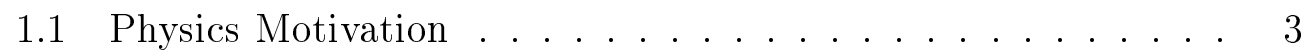

1.2 Design Goals . . . . . . . . . . . . . . . . . 4

2 Sign-Selected-Quadrupole-Train 5

2.1 Proton Beam Extraction . . . . . . . . . . . . 5

2.2 SSQT Alignment ..................... 6

3 Primary Proton Beam $\quad 9$

3.1 Proton Intensity Monitors . . . . . . . . . . . . . 10

3.2 Proton Dump Temperature Measurements . . . . . . . . 13

3.3 Beam Position Monitors (BPM's) . . . . . . . . . . 15

3.4 Vacuum SWIC's . . . . . . . . . . . . . 17

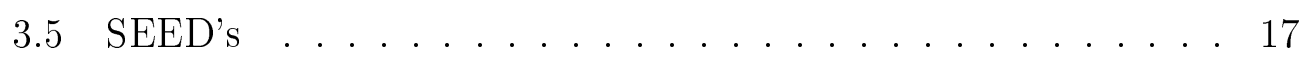

3.6 Results of Primary Beam Monitoring . . . . . . . . . 19

3.7 Total Number of Protons on Target . . . . . . . . . . . 20

4 Secondary Beam $\quad 21$

5 Neutrino Fluxes and Wrong Sign Contamination 22

5.1 Muon Neutrino Flux . . . . . . . . . . . . . . 23

5.2 Electron Neutrino Flux . . . . . . . . . . . 25

5.3 Wrong Sign $\nu$ Contamination ............ 26

6 Conclusions $\quad 28$

$\begin{array}{llr}7 & \text { Acknowledgements } & 28\end{array}$ 


\section{List of Figures}

1 Layout of the SSQT in N01 . . . . . . . . . . . 5 5

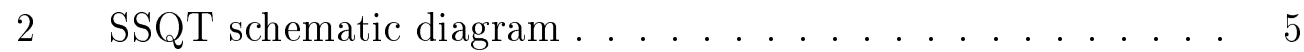

3 Schematic of SSQT with the elements used in $150 \mathrm{GeV}$ run . . 6

4 Data from the $150 \mathrm{GeV}$ run ............. . . 7

5 Results from the $150 \mathrm{GeV}$ run .............. 8

6 N01 pre-target monitor configuration . . . . . . . . . . . 9

7 NC1SEM intensity vs Tevatron intensity . . . . . . . . 10

8 Radiation damage of NC1SEM . . . . . . . . . . . 11

9 Intensities of the five pings as measured by beam toroid . . . . 12

10 Typical shape of a ping . . . . . . . . . . . . . 12

11 A schematic drawing of the proton dumps ........ 13

12 Dump Al temperature vs proton intensity . . . . . . . . 14

13 Proton intensity and position in a BPM . . . . . . . 15

14 BPM vs SEED positions. . . . . . . . . . . . 16

15 BPM vs SEED positions. . . . . . . . . . . . . 17

16 SEED horizontal beam profile . . . . . . . . . 18

17 Targeting positions and angles . . . . . . . . . . 20

18 Tertiary beam profiles as measured by the NW2 SWIC . . . . 21

19 Tertiary beam profiles as measured by the NW4 SWIC . . . . 22

20 Observed $E_{\nu}$ distributions of $\nu_{\mu}$ flux samples . . . . . . . . 23

21 The flux tuning procedure for $E_{\nu} \ldots \ldots \ldots 24$

22 The flux tuning procedure for beam position . . . . . . 25

23 Wrong sign muon $E_{\nu}$ distributions . . . . . . . . . . 27

24 Wrong sign muon $y$ distributions . . . . . . . . . 27

\section{List of Tables}

1 Targeting positions and angles . . . . . . . . . . 19

2 Total number of protons on target . . . . . . . . . . 20

3 Wrong sign muon contamination ........... 26 


\section{Introduction}

The weak mixing angle, $\sin ^{2} \theta_{W}$, is one of the fundamental parameters in the electroweak sector of the Standard Model (SM). Neutrino-nucleon deep inelastic scattering experiments provide an excellent test of the theory due to their high precision and different ranges of momentum transfer $\left(Q^{2}\right)$ compared to other experiments. CCFR (E770), the previous incarnation of $\mathrm{NuTeV}$ (E815), used the ratio of the cross sections of neutral current (NC) to charged current (CC) interactions to measure $\sin ^{2} \theta_{W}$, using the following Llewellyn-Smith formula [1] :

$$
R^{\nu(\bar{\nu})}=\frac{\sigma_{N C}^{\nu(\bar{\nu})}}{\sigma_{C C}^{\nu(\bar{\nu})}}=\rho^{2}\left(\frac{1}{2}-\sin ^{2} \theta_{W}+\frac{5}{9} \sin ^{4} \theta_{W}\left(1+\frac{\sigma_{C C}^{\bar{\nu}(\nu)}}{\sigma_{C C}^{\nu(\bar{\nu})}}\right)\right) .
$$

By assuming the SM expectation for $\rho[2]$ and knowing the fractions of $\nu$ and $\bar{\nu}$ 's in the beam, $\sin ^{2} \theta_{W}$ was extracted.

Using this method, however, resulted in large experimental systematic uncertainties. In order to reduce these uncertainties, E815 proposed a modification of the Neutrino Center (NC) beamline so that one sign of neutrino (either $\nu$ or $\bar{\nu}$ ) can be selected[3]. The sign selection of neutrinos enabled the experiment to use the Paschos-Wolfenstein equation[4]:

$$
R^{-}=\frac{\sigma_{N C}^{\nu}-\sigma_{N C}^{\bar{\nu}}}{\sigma_{C C}^{\nu}-\sigma_{C C}^{\bar{\nu}}}=\rho^{2}\left(\frac{1}{2}-\sin ^{2} \theta_{W}\right),
$$

which minimizes the largest uncertainties in $\sin ^{2} \theta_{W}$, which are caused by scattering from sea quarks and higher twist contributions to the cross sections. Two vertical bends, utilizing dipoles after the target, were introduced in the secondary beamline so that the sign selection was possible. At the same time, this reduced the electron neutrino $\left(\nu_{e}\right)$ flux resulting from $K_{L}$ decays, which was the second largest uncertainty in the CCFR analysis.

In following section, we discuss the physics motivation of the original proposal of the sign-selected-quadrupole-train (SSQT) and the design goal of the beamline.

\section{$1.1 \quad$ Physics Motivation}

In the previous measurement of $\sin ^{2} \theta_{W}$, the significant systematic uncertainties came from two major sources : 1) the model dependence of the final state charm quark production due to the mass threshold effect and 2) contamination of $\nu_{e}$-induced CC events to the $\nu_{\mu}$-induced NC sample. Since CC interactions of $\nu_{e}$ 's result in an electron and a hadronic shower in the final state, the electron shower gets lost in the hadronic shower and mimics exactly the topology of a $\nu_{\mu}$-induced $\mathrm{NC}$ event with no final state muon. 
In order to minimize the two major systematic uncertainties, two conditions are necessary : 1) a method that does not depend significantly on the final state charm production model and 2) significant reduction of the $\nu_{e}$ sources, especially from $K_{L}$ whose production cross section is known to only $20 \%$.

The method proposed by Paschos and Wolfenstein[4] utilizes two ratios

$$
\begin{aligned}
& R^{\nu}=\frac{\sigma\left(\nu_{\mu}, N C\right)}{\sigma\left(\nu_{\mu}, C C\right)} \\
& R^{\bar{\nu}}=\frac{\sigma\left(\bar{\nu}_{\mu}, N C\right)}{\sigma\left(\bar{\nu}_{\mu}, C C\right)}
\end{aligned}
$$

While in CC events, one can distinguish $\nu_{\mu}$ and $\bar{\nu}_{\mu}$ by the charge of the final state prompt muons, it is not possible at all to distinguish between $\nu_{\mu}$ and $\bar{\nu}_{\mu}$-induced events in the $\mathrm{NC}$ interactions, because they result in hadronic showers and corresponding neutrinos in the final state. Therefore, measurements of these two ratios can only be possible by separating the beam into neutrinos and anti-neutrinos.

Since Eq. 3 and 4 can be expressed in terms of $\rho$ and $\sin ^{2} \theta_{W}$ as in Eq. 1, E815 can determine both the unknown parameters, $\sin ^{2} \theta_{W}$ and $\rho$. At the same time, E815 can also perform the first measurements of the quantity:

$$
R^{-}=\frac{\sigma\left(\nu_{\mu}, N C\right)-\sigma\left(\bar{\nu}_{\mu}, N C\right)}{\sigma\left(\nu_{\mu}, C C\right)-\sigma\left(\bar{\nu}_{\mu}, C C\right)}
$$

without detailed knowledge of absolute cross sections, because dividing both the numerator and denominator in Eq. 5 by $\sigma\left(\nu_{\mu}, C C\right)$ results in:

$$
R^{-}=\frac{R^{\nu}-r R^{\bar{\nu}}}{1-r}
$$

where

$$
r=\frac{\sigma\left(\bar{\nu}_{\mu}, C C\right)}{\sigma\left(\nu_{\mu}, C C\right)}
$$

The quantity $r$ has been measured in E744 and E770[5], which can be used in the final $\sin ^{2} \theta_{W}$ analyses of E815.

\subsection{Design Goals}

Introducing vertical bends in the secondary beamline selects the secondary particles of the desired charge and dumps wrong sign and neutral particles, in particular the $K_{L}$ particles, to minimize the uncertainty in the $\nu_{e}$ contamination and the number of wrong sign neutrinos in the resulting neutrino beam. Achieving this provides a dramatic reduction in experimental systematic uncertainties, which should enable E815 to measure $\sin ^{2} \theta_{W}$ to an uncertainty of 0.003 and $\rho$ to 0.01 . 


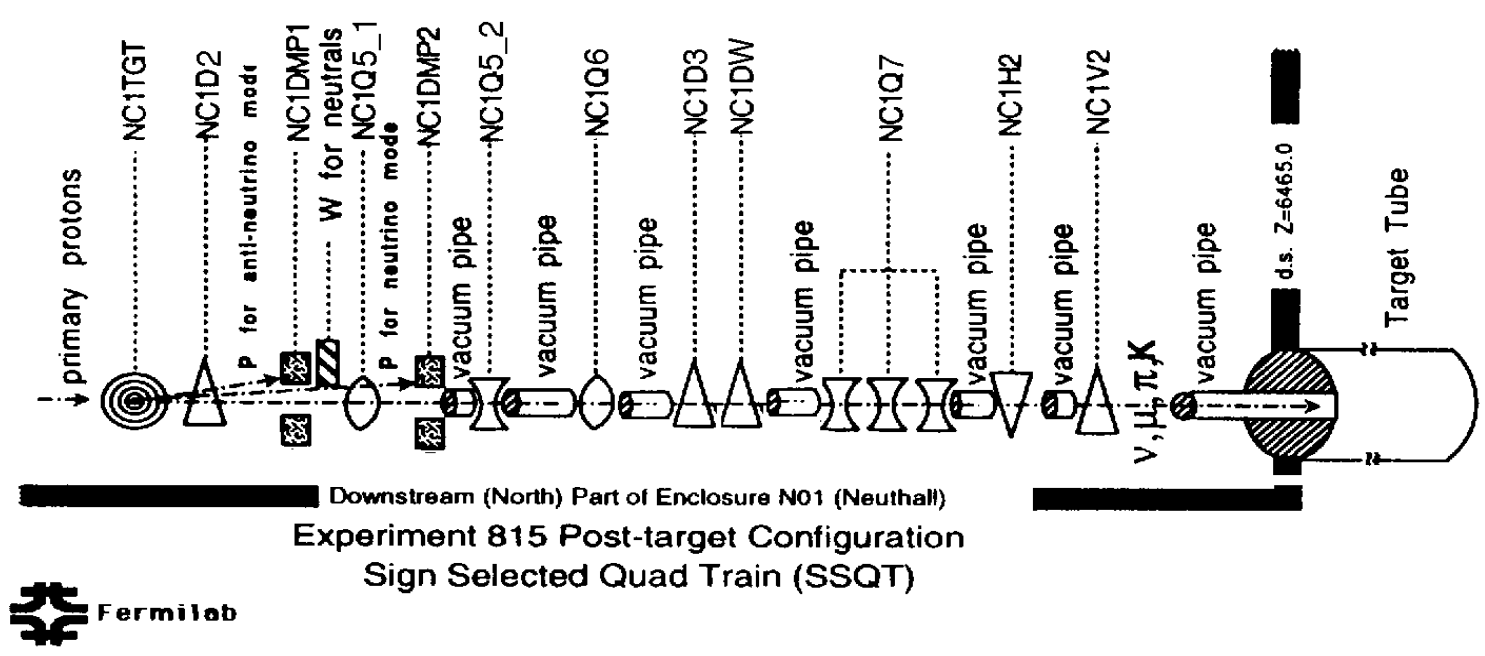

Figure 1: The layout of the SSQT in the enclosure N01.

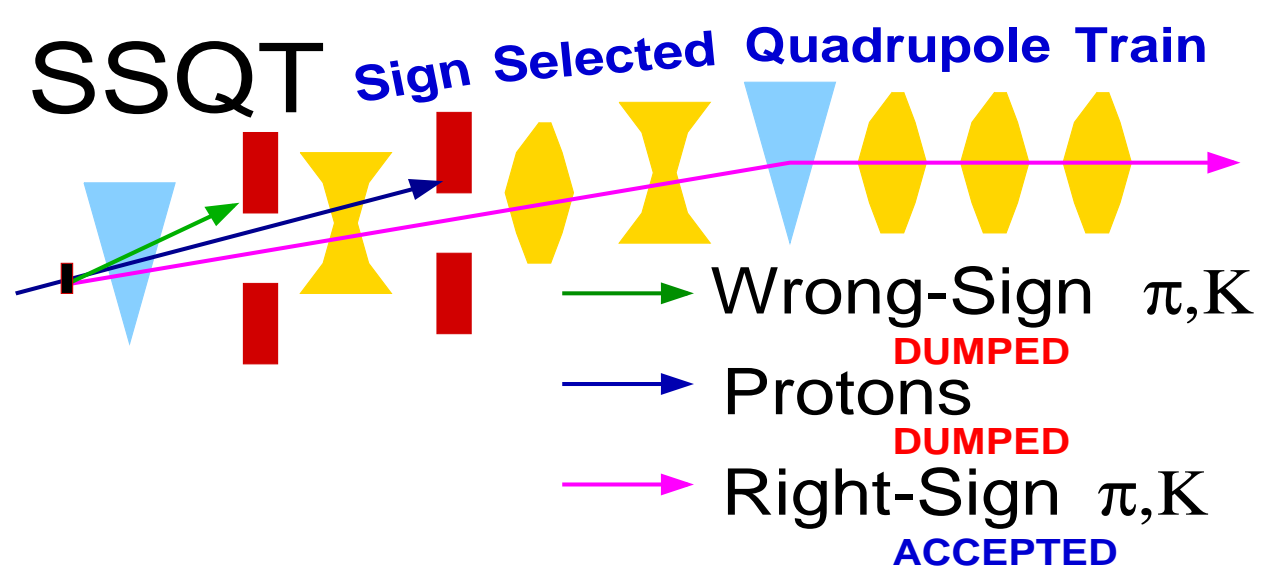

Figure 2: A schematic diagram of the SSQT optics.

\section{Sign-Selected-Quadrupole-Train}

In this section, general issues of the SSQT during the 1996-97 fixed target run are discussed. Figure 1 shows the SSQT optics, including the proper names of the beamline elements. Figure 2 shows the basic concept of the SSQT optics, illustrating the vertical bends and the dumping schemes of the remnant protons and wrong sign secondaries.

\subsection{Proton Beam Extraction}

At the beginning of the 1996-1997 fixed target run period, many different fast resonant proton extraction schemes were attempted in order to optimize the performance of the Tevatron, meeting the requirements of all the fixed target experiments. The final configuration was set such that there were five fast resonant extractions (pings) at the beginning of an approximately 1 


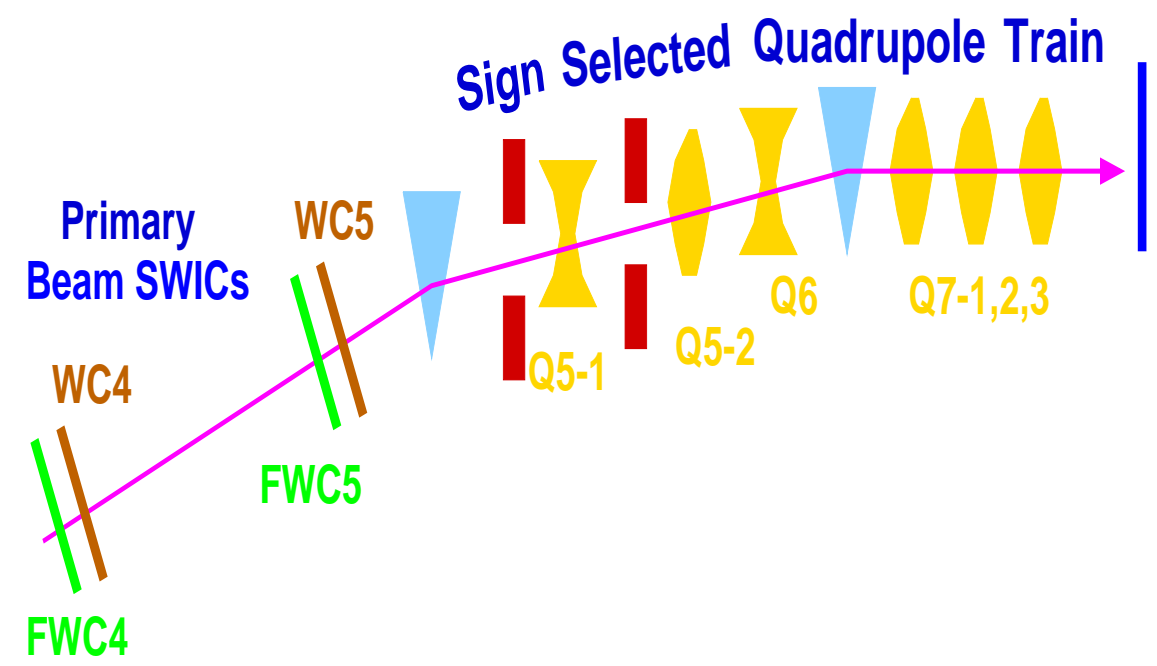

Figure 3: Schematic of SSQT with the elements used in $150 \mathrm{GeV}$ run. Shown are the two telescopes of primary beam SWIC's, FWC4\&5 and WC4\&5 and the six quadrupoles to be measured (see section 3.4 for more details).

minute long accelerator cycle, with a maximum ping intensity of $\sim 2 \times 10^{12}$ protons-on-target $(\mathrm{PoT}) / \mathrm{ping}$, with a 4 msec ping duration separated by 0.5 seconds, and a total intensity for the given cycle not exceeding $2 \times 10^{13} \mathrm{PoT}$ (see Figure 9).

The proton beam was incident on a 1 interaction length long BeO target, stationed at DUSAF $3460 \mathrm{ft}$, and the beam had a $7.8 \mathrm{mrad}$ upward angle relative to the horizontal axis pointing to the NuTeV detector. The secondary charged particles with the desired charge from the target were bent downward by $6.1 \mathrm{mrad}$ by the B-1 dipole (NC1D2) immediately downstream of the target. The currents of the secondary magnets were set so that the centerline of the beam is the path of the $250 \mathrm{GeV} / \mathrm{c}$ daughter mesons. The remaining $1.6 \mathrm{mrad}$ downward bend toward the $\mathrm{NuTeV}$ detector was provided by another B-1 dipole NC1D3 (see Fig. 1).

The two proton dumps were configured differently depending on the mode in which the experiment was run. The downstream dump (neutrino dump) did not move at all during the run while the upstream dump (anti-neutrino dump) moved in closer to the beam center line during the anti-neutrino mode running.

\subsection{SSQT Alignment}

In order to ensure proper alignment of the SSQT with respect to the incident proton beam, NuTeV proposed and carried out a $150 \mathrm{GeV}$ proton alignment run at the beginning of the 1996-1997 fixed-target run. The primary goal for this run was the alignment of the quadrupole magnets, which 


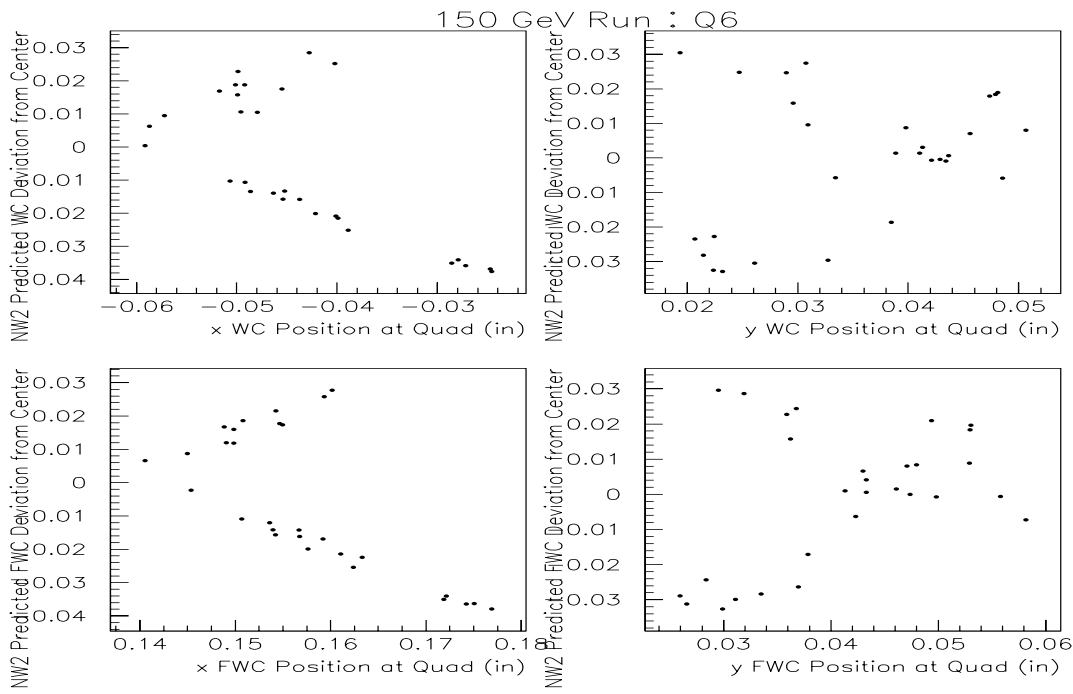

Figure 4: Data from the $150 \mathrm{GeV}$ run. Shown as a function of deviation from the beam-centerline is the deflection in inches at the NW2 SWIC, corrected for the absolute value of the magnetic field. (The two bands represent results from opposite polarities.) Where the two bands cross (zero deflection in a field) is the quadrupole center.

were the most crucial optical elements in the SSQT. NuTeV beam simulation required a 30 mil tolerance for the high field quadrupoles (NC1Q5 and NC1Q6) and 60 mil tolerance for the low field quadrupoles (NC1Q7_1, 22, and NC1Q7_3)[6].

A straight-through charged particle beam provided the most systematic alignment of the SSQT beam and quadrupoles available. The technique was to pass the beam along the nominal centerline, observe its position in a downstream segmented wire ion chamber (SWIC), and then turn on each quadrupole magnet in turn. Shifted quadrupole magnets act like the sum of a quadrupole and dipole field and thus deflect the beam position observed downstream of the quadrupole. Deviations in inches were measured as a function of distance from the nominal beam centerline as shown in Figure 4. Details of the run plan and execution can be found elsewhere[7]. A schematic of the key elements of the $150 \mathrm{GeV}$ run, the upstream SWIC's used to measure the initial trajectory of the primary beam, the quadrupoles, and the downstream SWIC's used to measure the deflection are shown in Figure 3.

The run was accomplished in two nights (with approximately 4 hours of beam each night) of $150 \mathrm{GeV}$ proton beam to the SSQT. The first night's data was difficult to interpret because the initial alignment of the beam with respect to the quadrupoles was far off-center. This turned out to be the result of two 50 mil misalignments of opposite signs of the FWC SWIC's with respect to the WC SWIC's. It should be noted that the required tolerance on 


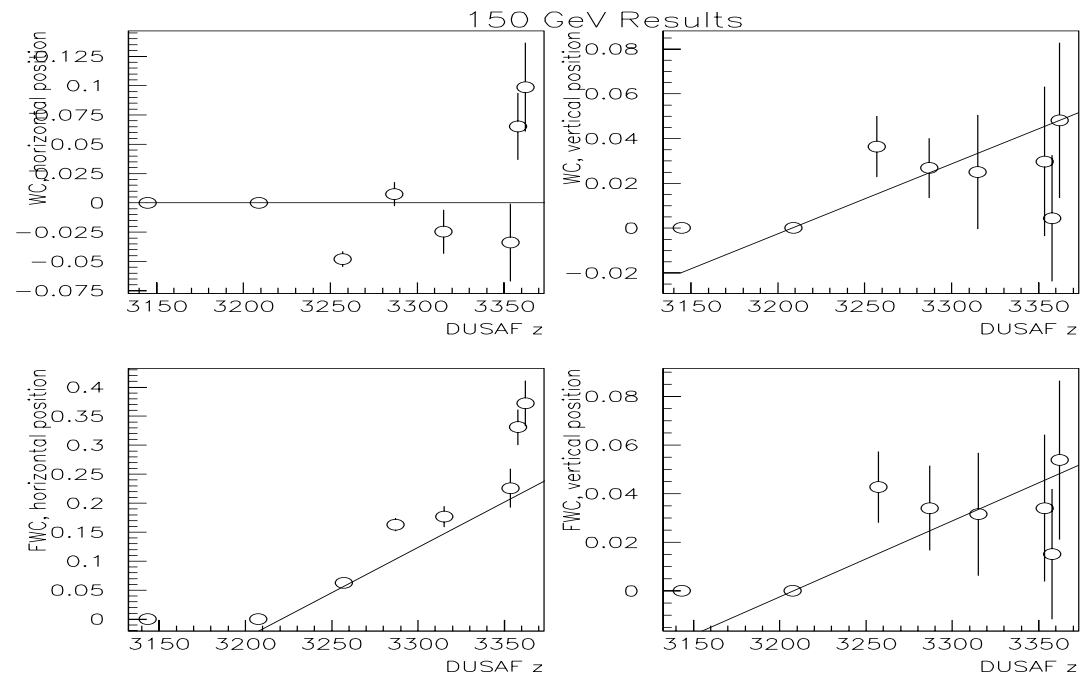

Figure 5: Resulting data from the $150 \mathrm{GeV}$ run. The measured quadrupole offsets in inches are shown as a function of the DUSAF $z$ position of the quadrupole with respect to beam centerlines established by the indicated SWIC telescope (FWC or WC). The measurement error includes systematics from differing magnetic fields and deflections from the nominal center. Uncertainties on quadrupole positions are well below tolerances.

the position of each of these SWIC's was 20 mils; had not the $150 \mathrm{GeV}$ run taken place, we would not have known which SWIC telescope was correctly aligned with respect to the beam, and uncertainties would have exceeded the tolerances of our beam simulation by a large factor.

The data taken with the beam far off-center in the quadrupoles provided weak evidence that the three downstream quadrupoles $(\mathrm{Q} 7-1,2,3)$ were mis-aligned by 90 mils with respect to the beam centerline. Therefore, between the two runs, survey and alignment crews repositioned these three quadrupoles.

The results of the second set of data is summarized in Figure 5. The positions of all six quadrupoles were measured to accuracies significantly better than the tolerances required by our beam Monte Carlo. Since the absolute offsets themselves were outside of our tolerances in the first night's measurement and gave us a chance to reposition the magnets, we conclude that the $150 \mathrm{GeV}$ was crucial for the accuracy of the NuTeV beam Monte Carlo and was successful. 


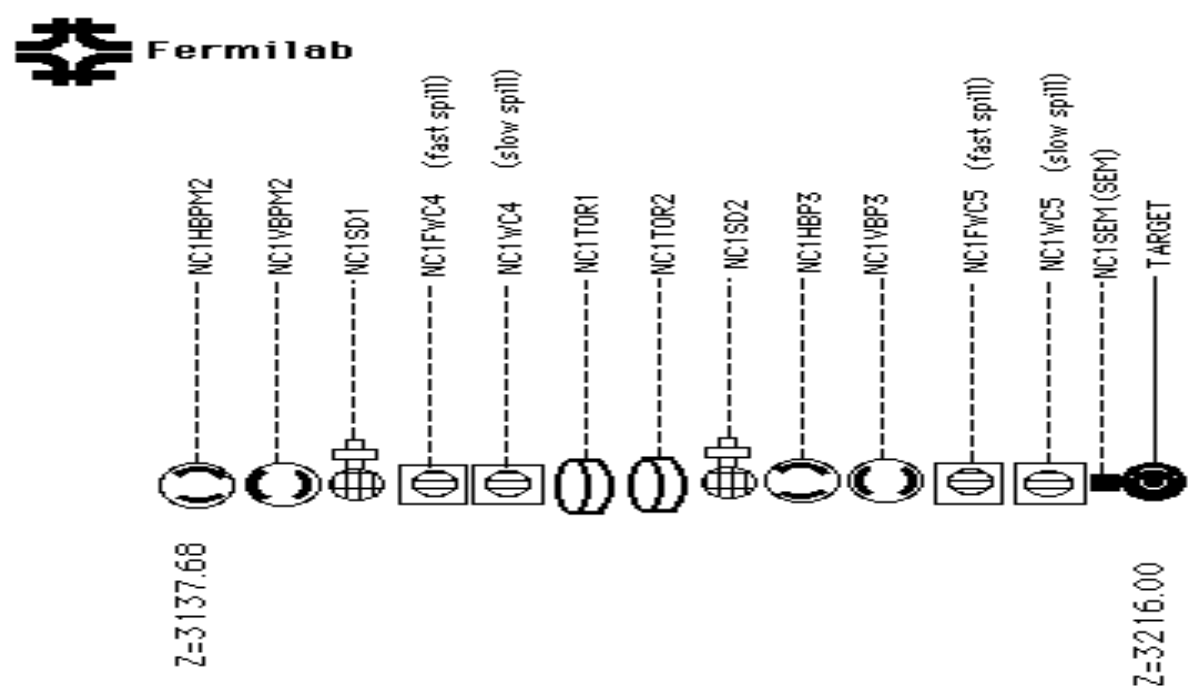

Figure 6: N01 pre-target monitoring configurations.

\section{Primary Proton Beam}

Primary beam monitoring is an important input in the beam simulation. Figure 6 shows the pre-target monitoring device configuration in N01, along with each device's proper name. The entire section shown in Figure 6 was maintained under very high vacuum, less than $10^{-3}$ Torr, in order for the monitors to function properly.

The size and shape of the primary proton beam spot affects the $K^{ \pm}$ spectrum[3]. The study performed for TM-1884[3] varied the spot size from a $\delta$-function to a Gaussian with $\sigma_{x}=0.7 \mathrm{~mm}$ and $\sigma_{y}=3 \mathrm{~mm}$ with the same center which showed essentially no differences in resulting $K^{ \pm}$induced neutrino energy distributions.

The targeting position and angle affect neutrino flux, mean energy, and beam positions at the detector. For instance, a targeting position deviation of 300 mils in either the horizontal or vertical axes results in $\sim 1 \%$ loss of the $\nu_{\mu}$ flux (see reference [6]). Thus, it is very important to know the positions and angles of the primary protons on target and incorporating the measurements into the beam Monte Carlo. In fact, the measurements of the primary beam positions and angles are being used as the direct inputs to the $\mathrm{NuTeV}$ beam Monte Carlo in estimating neutrino fluxes from $\pi^{ \pm}$and $K^{ \pm}$decays and other sources.

In this section, we discuss the primary beam monitoring devices and their performances, represented by the measurements of various primary beam parameters. 


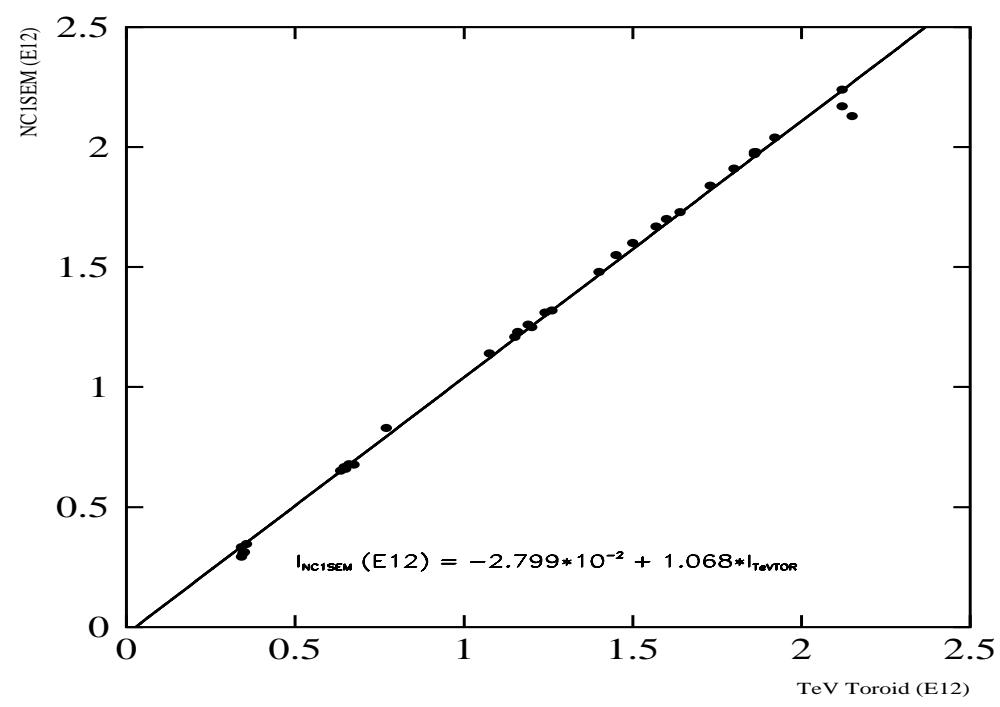

Figure 7: NC1SEM calibration constants. The intensity measured by the NC1SEM was calibrated relative to the Tevatron intensity measured by the TeV toroid.

\subsection{Proton Intensity Monitors}

The intensities of the proton beam in the pings were measured by four independent devices; 1) a standard thin foil SEM located immediately upstream of the BeO target, 2) a beam current toroid, 3) Beam Position Monitors (BPM's), and 4) Secondary Emission Electron Detectors (SEED's) which were also used to monitor the beam position and targeting angles. Since the BPM's and SEED's are discussed in the beam position monitoring section, we will concentrate on the SEM and beam toroid primary intensity measurements in this section.

A secondary emission monitor (NC1SEM) was located immediately upstream of the target to measure the proton intensity. NC1SEM was a standard thin foil SEM and was calibrated in coordination with the Beams Division early in the run. Figure 7 shows the SEM calibration constants measured relative to the Tevatron toroid intensity. The calibration was performed by spilling out all the protons in the Tevatron into the NC beamline, assuming the loss in transporting the beam is negligible. The line in Fig. 7 is a linear fit of the NC1SEM intensity $\left(I_{N C 1 S E M}\right)$ relative to the Tevatron intensity as measured by the Tevatron toroid $\left(I_{T e V-T o r}\right)$.

During the run period, it was found that the thin foil SEM was damaged from the radiation due to the high proton intensity passing through the foil. Figure 8 shows the progressive degradation of NC1SEM by showing the variations of the NC1SEM calibration constant relative to the $\mathrm{NuTeV}$ beam 


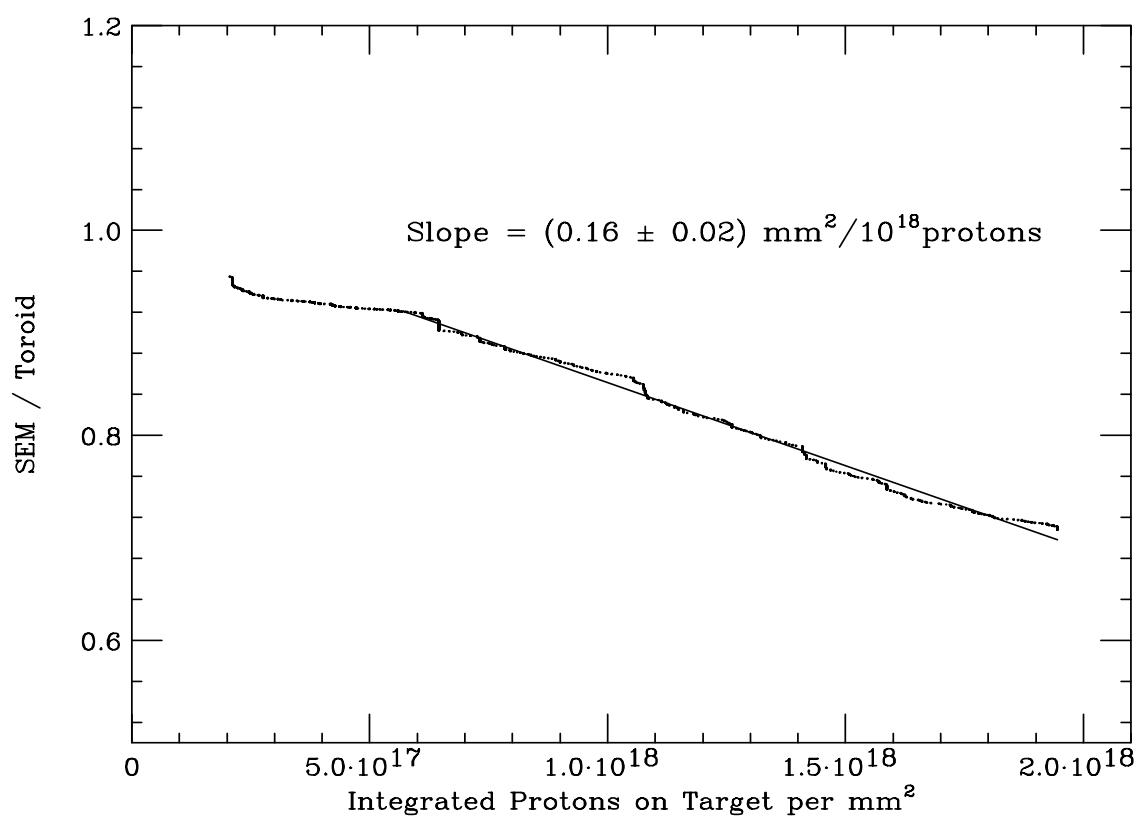

Figure 8: NC1SEM relative calibration constants as a function of integrated protons intensity per $\mathrm{mm}^{2}$. The $\mathrm{NuTeV}$ beam toroid was used as the measure of the true intensity.

toroid as a function of total integrated proton intensity per unit area $\left(\mathrm{mm}^{2}\right)$. The line in the figure represents the linear fit on the last $2 / 3$ of data with a slope of $(0.16 \pm 0.02) / 10^{18}$ protons $/ \mathrm{mm}^{2}$, because the beam was known to be stable in both position and profile during this period.

In addition to NC1SEM, two beam toroids were installed in the pre-target beamline in order to measure the primary proton beam intensity accurately. Only one of them was used during the run, while the other one was a spare. Figure 9 shows the intensities of all five pings as a function of time since the beginning of the ping.

Figure 10 shows the typical shapes of pings at two different times during the run, as measured by the beam toroid. The gaussian shape of the ping, shown in the solid histogram, caused a $\sim 3 \%$ detector dead time due to a high concentration of neutrino events within a very short time ( $\sim 1 \mathrm{msec})$. $\mathrm{NuTeV}$ requested to the Beams Division to stretch out the shape of the pings to minimize the detector dead time caused by this concentration of neutrino events. The dashed histogram shows the shape of the ping after the change was made. The change in the ping time structure was achieved by modifying the Tevatron QXR current driving waveform from a linear to a more logarithmic shape as a function of time. A more detailed description of the modifications can be found in Ref.[8]. The resulting event rate at the detector was more evenly distributed throughout the ping, reducing the $\sim 3 \%$ detector dead time caused by the beam to less than $1 \%$. This effectively 


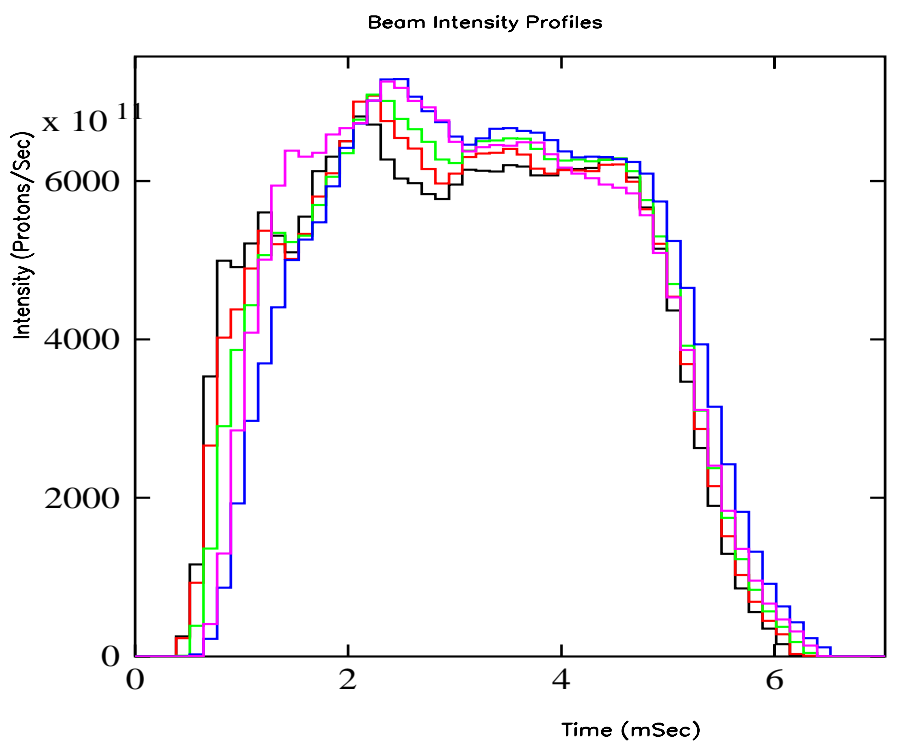

Figure 9: Proton intensities of the five pings as a function of time from the beginning of the pings measured in the $\mathrm{NuTeV}$ beam toroid.

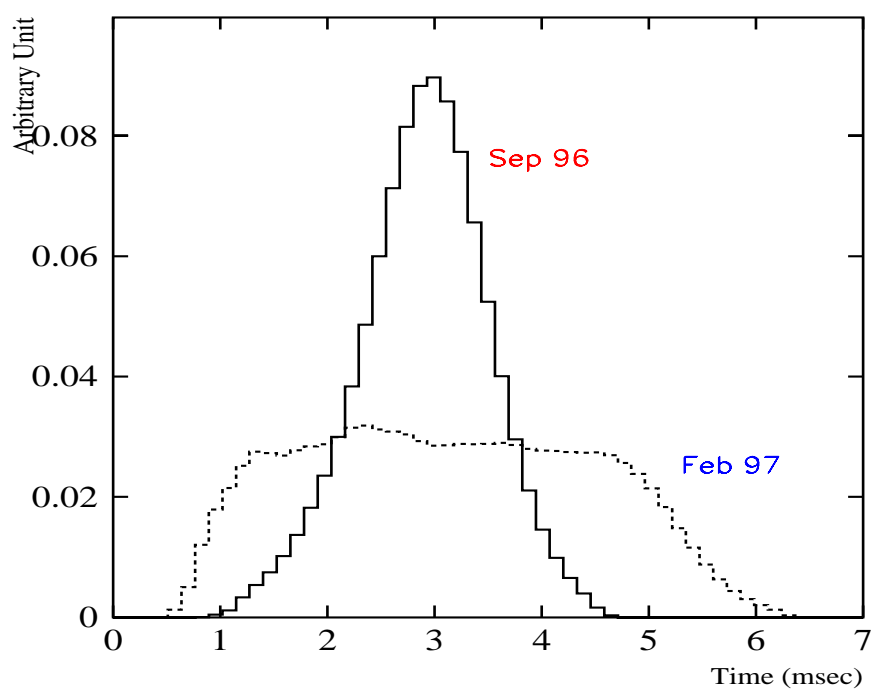

Figure 10: Typical shapes of a single ping in September 96 (solid) and February 97 (dashed), measured in the beam toroid. 


\section{Beam View of Dumps}
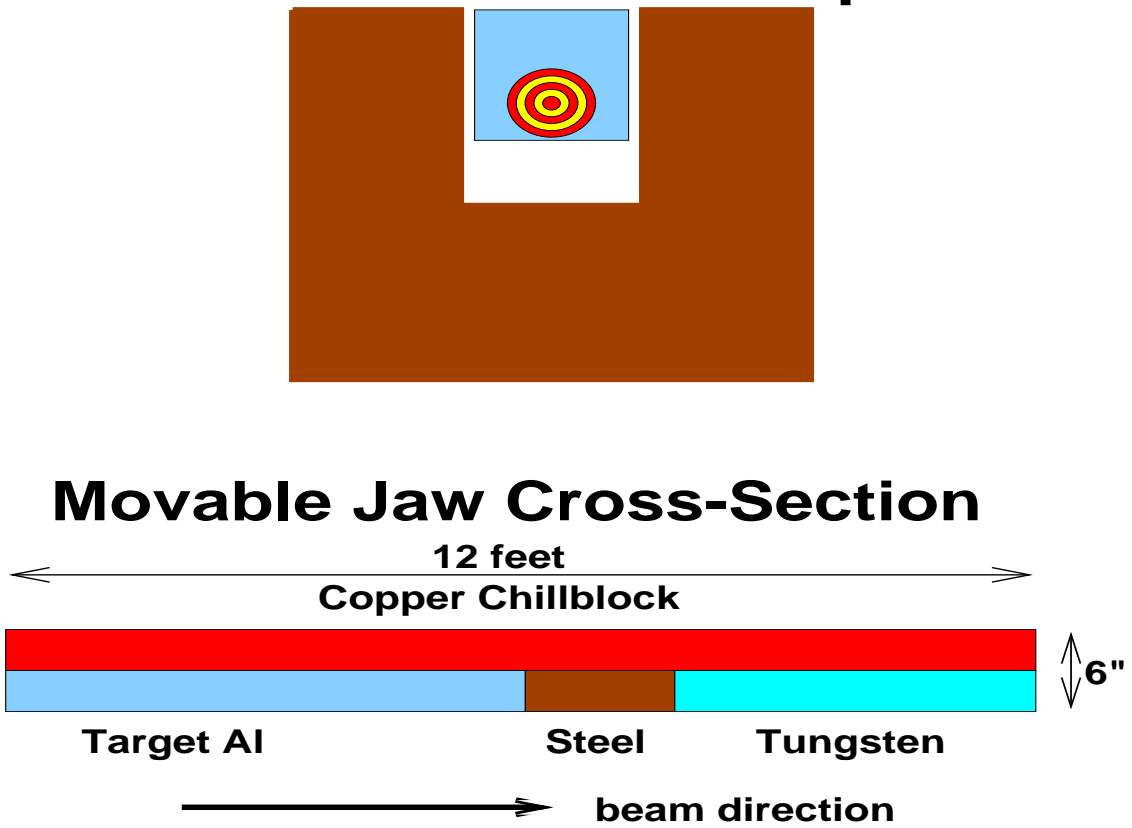

Figure 11: A schematic drawing of the proton dumps. The figure on the top shows the beam-view of the dump and the bottom shows the side view of the upper section of the dump.

gained $\sim 100$ hours of beam time for $\mathrm{NuTeV}$ over the course of the run.

The calibration of the $\mathrm{NuTeV}$ beam toroid was done in every cycle using a test pulser which passed a known amount of current through the toroid. This calibration was checked with the Tevatron single beam running against the Tevatron toroid intensity measurement $\left(I_{\text {Beam }}\right)$. The two intensities were the same to within $5 \%$.

\subsection{Proton Dump Temperature Measurements}

The remnant protons from the high intensity primary beam are dumped into two physically different proton dumps in the SSQT train, depending on the mode. The sizes and considerations of the dump structure were covered in TM-841 and TM-1884[3]. We discuss the effect of the remnant protons and the wrong sign secondaries in this section by presenting the dump temperature variations.

Since the effects of released energy differ in different sections of the dump due to the progression of the hadronic showers which are induced from interacting protons and wrong charge secondary particles, resistance temperature detectors (RTD's) were installed in various parts of the SSQT from the target to the downstream end of the second dump to monitor temperatures. Figure 11 shows a schematic drawing of the structure of both the proton 

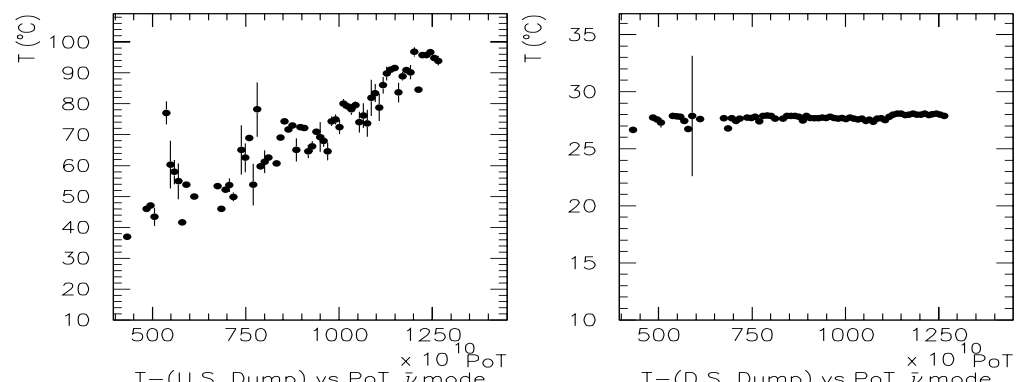

T-(U.S. Dump) vs POT, $\bar{\nu}$ mode
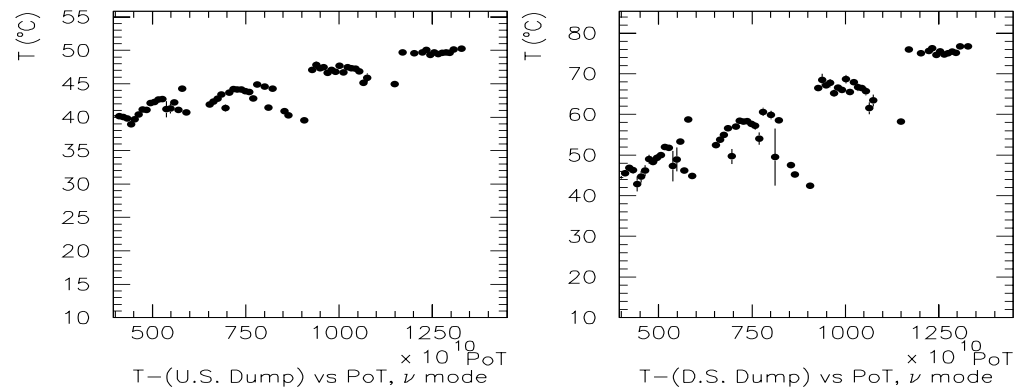

Figure 12: Temperature variation of the Al section of the upstream and downstream proton dumps for both neutrino and anti-neutrino modes.

dumps. The figure on the top shows the beam view of the dump and the bottom shows the cross-section of the upper section of the dump that the remnant protons hit. The beam enters from the left hitting the $6^{\prime} \mathrm{Al}$ section of the upper dump jaw.

Figure 12 shows the correlations between the intensity of the incident proton beam and the temperatures of the $6^{\prime} \mathrm{Al}$ sections of the dumps that the remnant proton beam and the wrong sign secondaries hit for neutrino and anti-neutrino modes. In anti-neutrino mode, the upstream (US) dump is moved in closer to the center of the beam to the distance of 0.62 " from the central ray trajectory to absorb remnant protons and wrong sign secondaries. Thus, the temperature of the US dump Al section increases almost linearly with the proton intensity, while the $\mathrm{Al}$ section of the downstream (DS) dump does not show any temperature variation.

On the other hand, in neutrino mode, the US dump is moved out to the distance of 1.85 " from the central ray trajectory absorbing only the wrong sign secondaries and the DS dump absorbs the remnant proton beam. The bottom two plots show the temperature variations of the same Al sections of the two dumps in neutrino mode. The US dump shows less dependence to the proton intensity, while it is apparent that the DS dump temperature displays steeper dependences on the proton intensity.

The intensities of the pings and the temperatures of various sections of the dumps were carefully monitored to prevent mechanical damage of the 


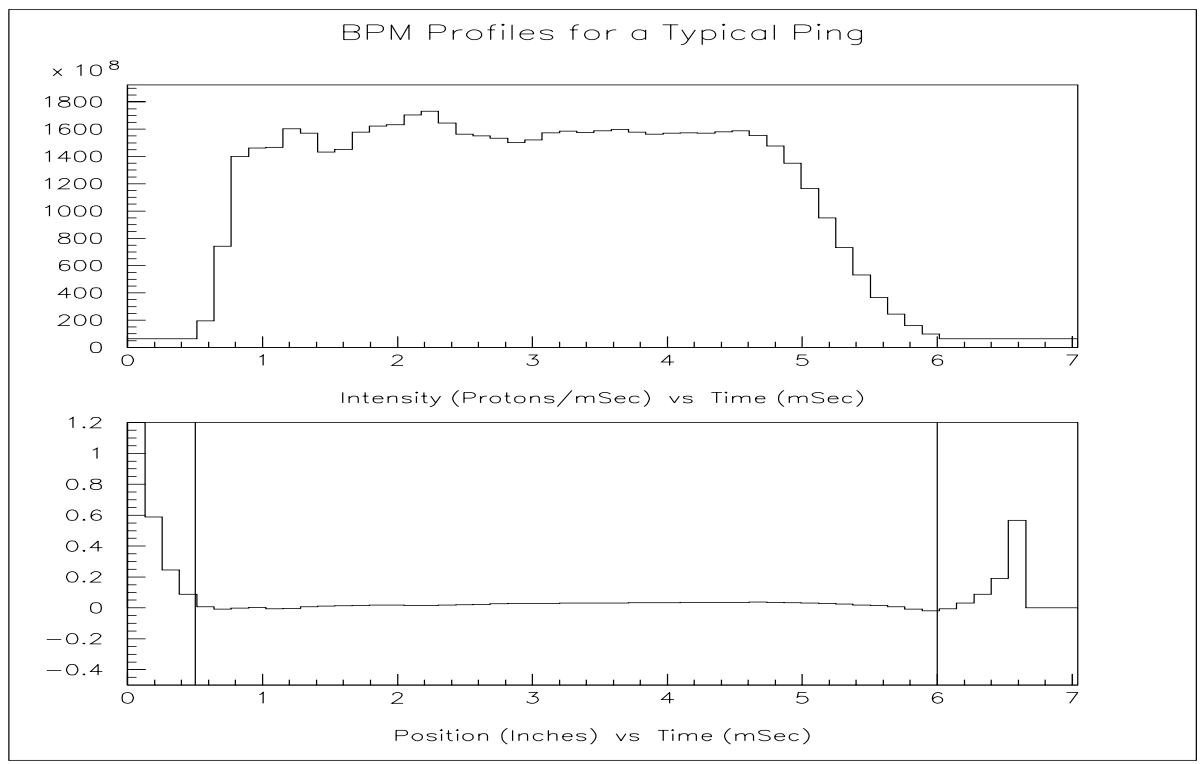

Figure 13: Intensity and the position of the proton beam as a function of time (ms) since the beginning of the ping, measured by a BPM.

dump. At no time over the course of the run was any section of the dump's temperature allowed to exceed $110^{\circ} \mathrm{C}$.

\subsection{Beam Position Monitors (BPM's)}

There were a total of four beam position monitors (BPM's) built by the University of Oregon group. Two of each of these were paired to monitor both horizontal and vertical beam positions. The two BPM's in a given pair were located immediately next to each other in order to minimize any possible systematic mis-measurements of the positions. One set of BPM's was located at the most upstream portion of the pre-target monitors and the other set was located just before the target. Position resolutions of these BPM's were measured to be $\sim 0.1 \mathrm{~mm}$.

Since the BPM's measure the current carried by the proton beam, we also configured the electronics to monitor proton intensity during the given fast ping as a function of time from the beginning of the ping. Figure 13 shows the intensity and beam position of protons in a single ping as a function of the time since the beginning of the ping. Both the intensity and position within the ping were stable to a few percent and to $\sim 0.06$ mils respectively throughout the run. Figure 15 shows the horizontal and vertical positions measured by the BPM's in the upstream pair (top) and by the downstream pair (bottom) compared to those measured by the corresponding SEED's. The BPM measured positions are in perfect correlations with the SEED's. 

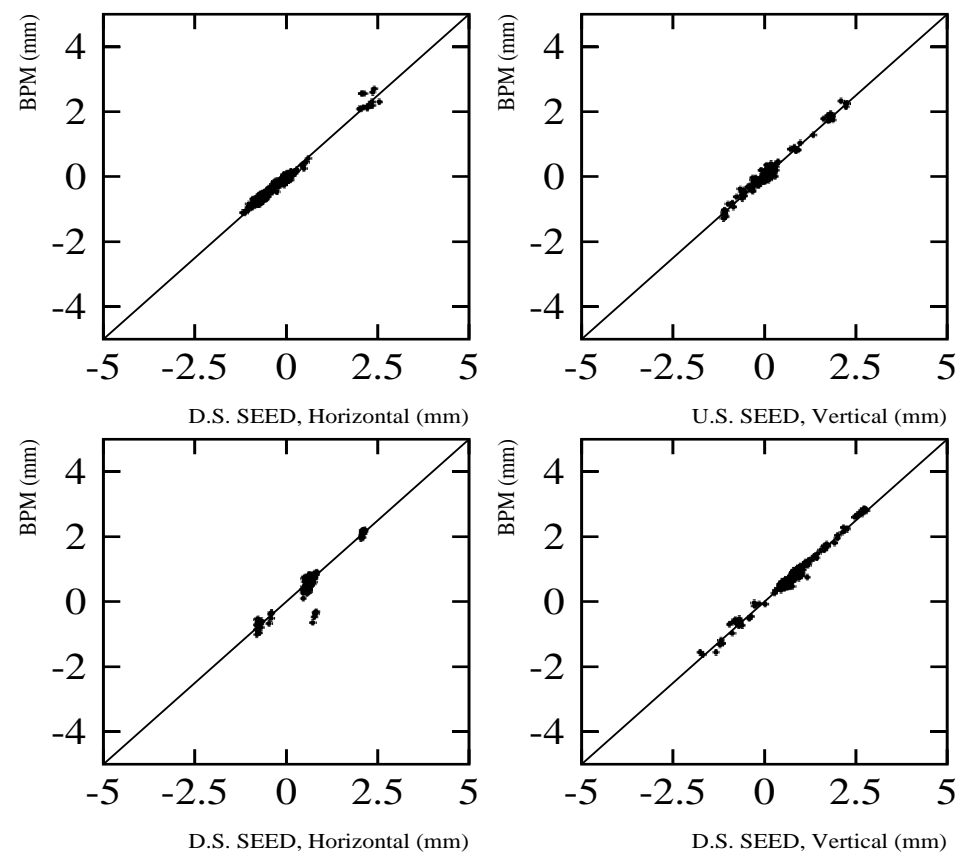

Figure 14: Correlations between the positions as measured by the BPM's versus as measured by the SEED's. The lines represent the perfect correlations.

\subsection{Vacuum SWIC's}

There were four vacuum SWIC's used in the pre-target $\mathrm{NC}$ beamline to monitor the primary proton beam positions and angles. Two were configured to monitor profiles during the fast spill (NC1FWC4 and NC1FWC5), which consisted of five consecutive pings of 4 msec separated by 0.5 sec to maximize duty factors, and an intensity of $\sim 2 \times 10^{12}$ protons per ping. The other two SWIC's were configured to monitor profiles during slow spill (NC1WC4 and NC1WC5) which followed the last fast ping by approximately a 1 second separation. These SWIC's were complementary to the SEED's and used mostly for beam tuning purposes.

The position resolution of these SWIC's was $120 \mu \mathrm{m}$ in both horizontal and vertical directions. Figure 16 shows the beam profiles measured by these SWIC's compared to the corresponding SEED's.

\subsection{SEED's}

The SEED's[9] provide accurate profiles and positions at small wire spacing $(125-500 \mu \mathrm{m})$ in a high energy, high rate environment that exceeds the capabilities of SWIC's. This device has been designed and constructed to 

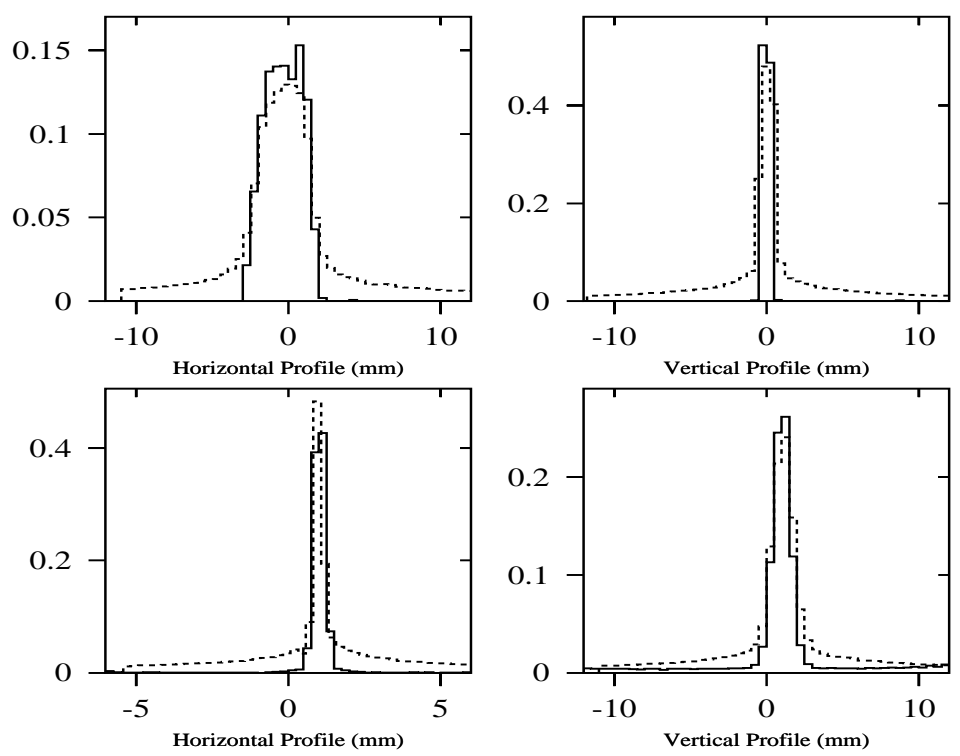

Figure 15: Comparisons of the horizontal and vertical beam profiles from the upstream (top) and downstream (bottom) SEED's (solid) and the corresponding vacuum SWIC's (dashed).

monitor the beam position and profile of the NC beamline, with an average beam width at the target of $\sigma=0.6 \mathrm{~mm}$ which is known to $10 \%$. NuTeV received $\sim 1 \times 10^{13} 800 \mathrm{GeV}$ protons in five pings per cycle throughout most of the 1996-1997 fixed target run.

Secondary emission detectors have been used at Fermilab and at other laboratories for measuring beam intensity, position and profile for many years[10, 11]. The SEED's were built to measure precisely the beam position and profile and can measure beam intensity by adding an intensity section to the detectors.

The NuTeV beam was measured at two stations before the target. At the upstream station the beam had a full width at half maximum (FWHM) of $2.6 \mathrm{~mm}$ horizontal and $1.9 \mathrm{~mm}$ vertical, and at the downstream station 0.7 $\mathrm{mm}$ horizontal and $2.4 \mathrm{~mm}$ vertical, meeting the requirements in Ref.[3].

The profile section contains $x$ and $y$ signal planes located between three bias foils. In order to satisfy the beamline requirements, two types of ceramic boards were designed: a single sided board with a wire pitch of $0.500 \mathrm{~mm}$ and a double sided board with a pitch of $0.250 \mathrm{~mm}$ and $0.125 \mathrm{~mm}$ offset between the front and back sides. A description of how the wires were soldered to the ceramic substrate is given in Ref.[12]. The detectors were placed in the beam only about $3 \%$ of the total run time, or for about $1 \times 10^{17}$ protons. The estimated resolution is $3.5 \mu \mathrm{m}$ for the $125 \mu \mathrm{m}$ boards, $7 \mu \mathrm{m}$ for the 
Table 1: Summary of the primary proton targeting positions and angles, relative to the center of the $\mathrm{NuTeV}$ target and to the horizontal line pointing to the detector, measured by the SWIC's and cross checked by the SEED's.

\begin{tabular}{ccc} 
Quantity & Mean & Width $(\mathrm{RMS})$ \\
\hline$x(\mathrm{~mm})$ & $9.54 \times 10^{-2}$ & 0.15 \\
$y(\mathrm{~mm})$ & $7.00 \times 10^{-2}$ & 0.38 \\
$\theta_{x}(\mathrm{mrad})$ & $6.23 \times 10^{-3}$ & $2.57 \times 10^{-2}$ \\
$\theta_{y}(\mathrm{mrad})$ & $-1.47 \times 10^{-2}$ & $1.70 \times 10^{-2}$
\end{tabular}

$250 \mu \mathrm{m}$ boards, and $14 \mu \mathrm{m}$ for the $500 \mu \mathrm{m}$ boards. The measured secondary electron efficiency is $(4.0 \pm 0.5) \%$ as compared to a current transformer. This is related to the expected number of electrons liberated per proton that hits a wire.

Figure 16 show comparisons between the SEED (solid histograms) and corresponding vacuum SWIC's (dashed histograms) at the upstream station (top two sets of histograms) and downstream station (bottom two sets) in the $\mathrm{NuTeV}$ beam for horizontal and vertical axes, respectively. Except in the tails, the two sets of profiles agree very well.

\subsection{Results of Primary Beam Monitoring}

Targeting angles of the primary proton beam were measured by three sets of independent devices upstream of the target, and these measurements are incorporated into the NuTeV beam Monte Carlo. Figure 17 shows the primary beam horizontal and vertical positions in $\mathrm{mm}$ and targeting angles in mrad in both the horizontal and vertical directions, measured by the monitoring devices (SWIC's and SEEDs) throughout the entire run period. The BPM's were not used to measure for these measurements, because the measured positions drifted in large time scale (more than an hour) due presumably to instability of the readout electronics. See reference [13] for more detailed discussion concerning the performance of BPM's. Table 1 summarizes the primary proton beam positions and the targeting angles throughout the entire run, measured by the primary fast SWICs (NC1FWC4 and FWC5) and cross checked by the SEED measurements.

The positions and angles are relative to the center of the $\mathrm{BeO}$ target and relative to the expected proton beam angle, respectively. The typical position offset from the center of the target was on the order of $8 \times 10^{-2} \mathrm{~mm}$ in both directions and the targeting angle offset was on the order of $2 \times 10^{-3} \mathrm{mrad}$. 


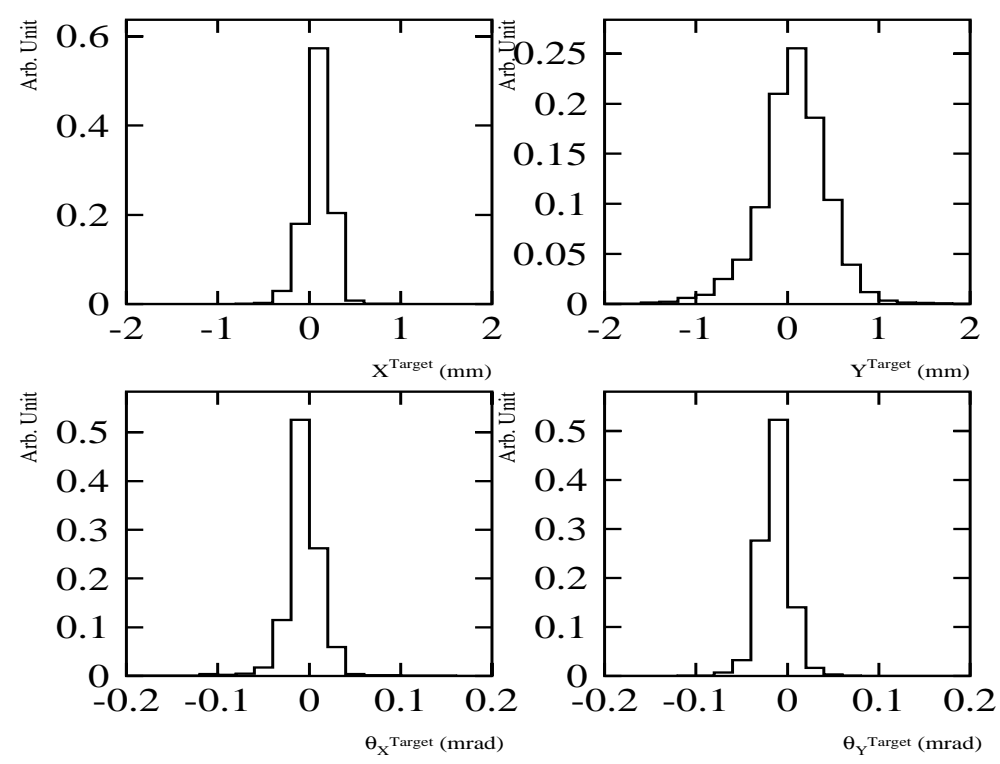

Figure 16: Targeting positions and angles of the primary protons as measured by the the pre-target SWIC's and cross checked by the SEED's.

\subsection{Total Number of Protons on Target}

E815 received a total integrated proton intensity of $3.15 \times 10^{18}$ through $1,557,204$ fast pings. The total live time of the $\mathrm{NuTeV}$ detector with beam was 2 hours and 13.5 minutes. Table 2 summarizes the integrated proton intensity for neutrino and anti-neutrino modes separately, along with corresponding detector live times.

The fractional running times for neutrino and anti-neutrino modes are $45.5 \%$ and $54.5 \%$. The data taking efficiencies of the $\mathrm{NuTeV}$ experiment are $92.7 \%$ and $96.3 \%$ for neutrino and anti-neutrino modes, respectively. The efficiencies are slightly different for neutrino and anti-neutrino modes

Table 2: List of the total number of protons on target, delivered, delivered with DAQ on, detector live, and detector live time fraction for neutrino and anti-neutrino modes.

\begin{tabular}{ccccc} 
Mode & Delivered & Delivered w/ DAQ & Recorded & Live $(\%)$ \\
\hline \hline Neutrino & $1.43 \times 10^{18}$ & $1.37 \times 10^{18}$ & $1.27 \times 10^{18}$ & 92.7 \\
Anti-Neutrino & $1.72 \times 10^{18}$ & $1.64 \times 10^{18}$ & $1.58 \times 10^{18}$ & 96.3 \\
\hline Total & $3.15 \times 10^{18}$ & $3.01 \times 10^{18}$ & $2.86 \times 10^{18}$ & 95.0
\end{tabular}



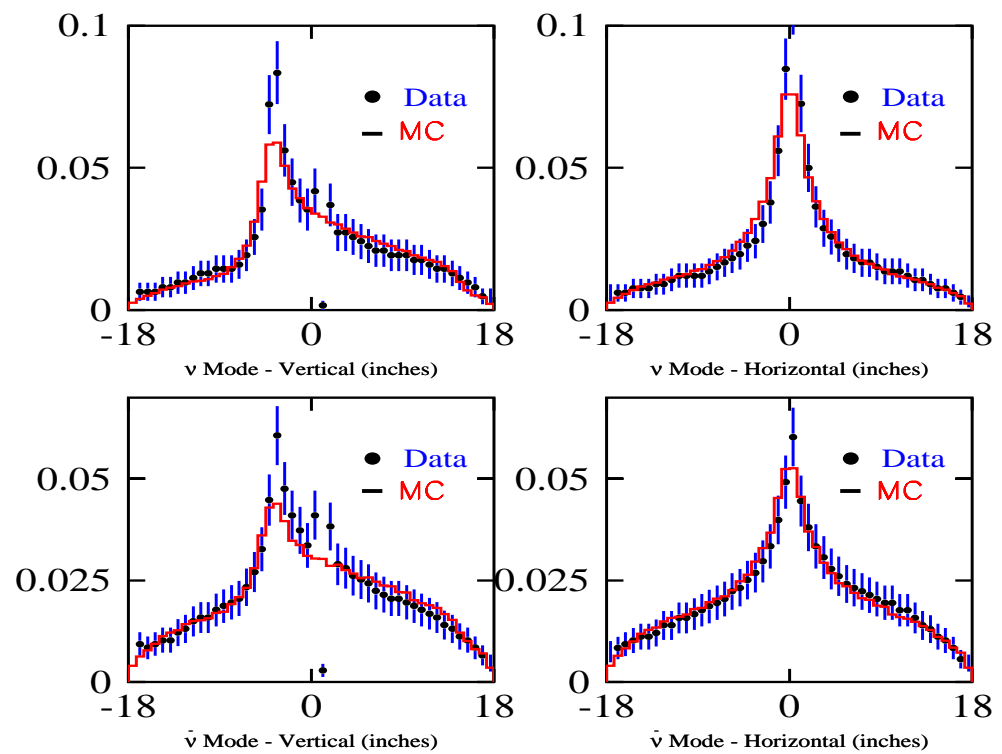

Figure 17: Beam profiles measured at the NW2 SWIC. Solid circles represent data and the histograms represent Monte Carlo predictions.

because the event rate in neutrino mode is about a factor of 5 bigger than that of the anti-neutrino mode. Less than $0.03 \%$ of the data was taken with spills of intensity less than $5 \times 10^{11} \mathrm{PoT}$.

\section{Secondary Beam}

Knowledge of the secondary beam center to $1 \mathrm{~cm}$ accuracy was quoted to suffice in TM-1884[3]. In this section, we discuss the measured profiles of the secondary beam in the tertiary SWIC's and compare the profiles with the NuTeV beam Monte Carlo.

There were two SWIC's, one each in the enclosures NW2 (NC2WC1) and NW4 (NC4WC1), in order to monitor the profiles of the tertiary beam. These SWIC's were used to assist in understanding the beam as well as to spot problems in the secondary $\pi$ and $K$ beams.

The NW2 SWIC[14] used a He-mixture $\left(98 \% \mathrm{He}\right.$ and $\left.2 \% \mathrm{H}_{2}\right)$ gas while the NW4 SWIC used the standard $\mathrm{ArCO}_{2}$ gas as the medium. As was proposed in the Ref.[3], NuTeV placed a large area SWIC in NW2 $(0.91 \mathrm{~m} \times 0.91 \mathrm{~m})$ immediately downstream of the vacuum window of the decay pipe and in NW4 $(1.83 \mathrm{~m} \times 1.83 \mathrm{~m})$ immediately behind the lead shield berm. Most of the particles leaving hits on the NW4 SWIC are expected to be muons, while the particles at NW2 SWIC are remaining un-decayed $\pi$ and $K$, along with 

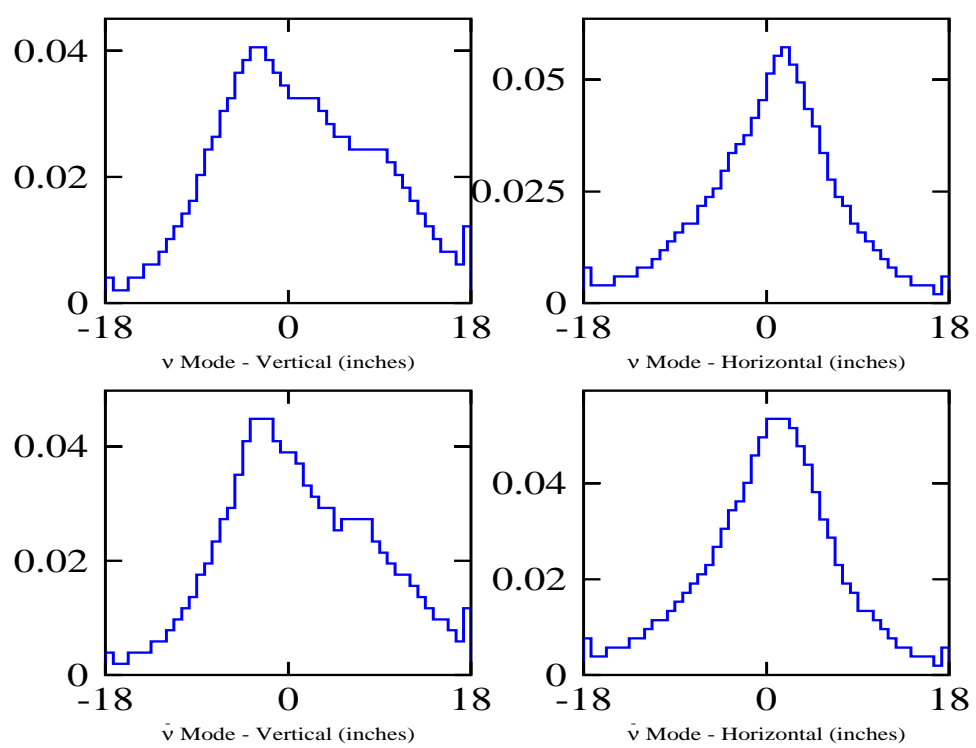

Figure 18: Tertiary beam profile measured at the NW4 SWIC in neutrino and anti-neutrino modes.

tertiary muons resulting from upstream $\pi$ and $K$ decays.

The resolutions of these SWIC's are better than $200 \mu \mathrm{m}$ and is much better than what was proposed in TM-1884. Figure 18 shows the NW2 SWIC profiles along the horizontal and vertical axes for neutrino (top) and anti-neutrino (bottom) modes. The solid points are data and the histograms are the predictions from the NuTev beam Monte Carlo, a simple toy Monte Carlo based on the decay Turtle with a flat background distribution added to match the tail. The profiles and the peak positions along both axes for both modes agree well with the predictions, except $1 \sim 1.5 \sigma$ deviation at the peak. Figure 19 shows the profiles measured by the NW4 SWIC. The profile is not yet compared to predictions. However, the good agreements of the NW2 SWIC profiles with the beam Monte Carlo give us confidence that we understand the beam well.

\section{Neutrino Fluxes and Wrong Sign Contam- ination}

The biggest considerations of the original SSQT proposal were the backgrounds. The two major background sources that were assessed in the TM1884 [3] were: 


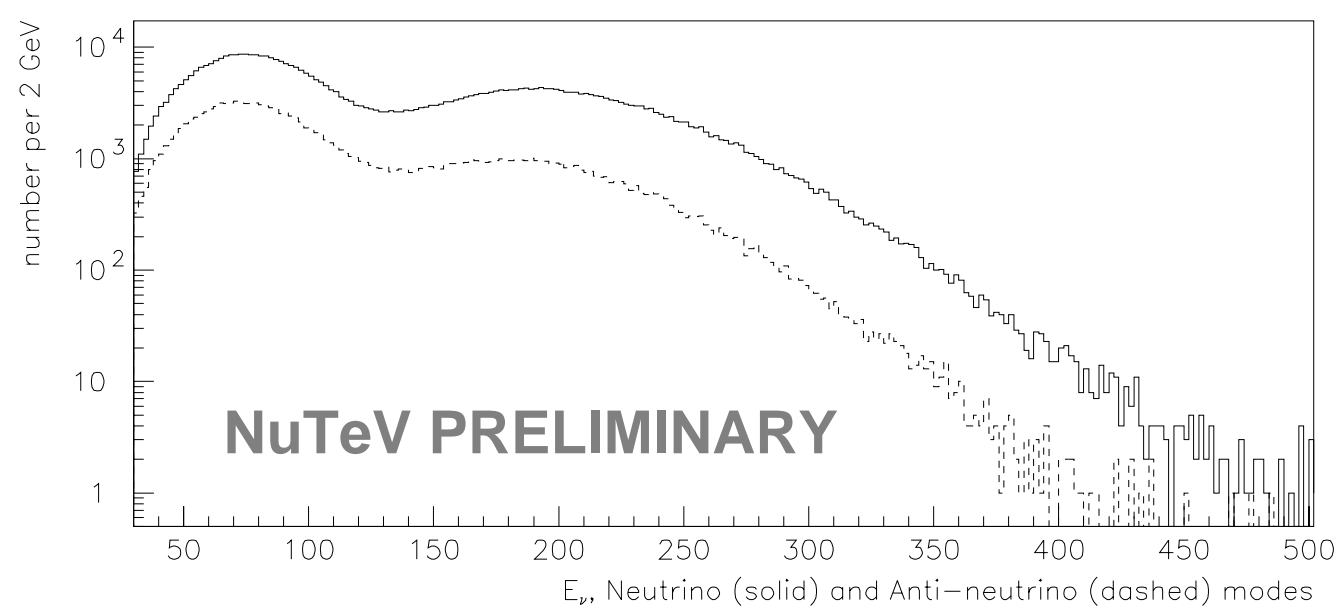

Figure 19: The number of events vs observed $E_{\nu}$ for the events in the muon neutrino flux tuning sample.

- $\nu_{e}$ production.

- Wrong sign backgrounds.

In this section, we discuss both issues and present the measured flux, using the information provided by our primary beam monitoring devices and the measured upper limits of the wrong sign neutrino contamination resulting from possible scraping of the beam from the secondary targets, such as vacuum flanges, beam pipes, vacuum windows, etc.

\subsection{Muon Neutrino Flux}

The NuTeV neutrino beam results primarily from the decay of sign-selected $\pi$ 's and K's in the decay region downstream of the SSQT. The dominant processes leading to $\nu_{\mu}\left(\bar{\nu}_{\mu}\right)$ in the positive (negative) focusing mode of the SSQT are:

$$
\begin{array}{lll}
\pi^{+} \rightarrow \nu_{\mu} \mu^{+} & K^{+} \rightarrow \nu_{\mu} \mu^{+} & K^{+} \rightarrow \pi^{0} \nu_{\mu} \mu^{+} \\
\left(\pi^{-} \rightarrow \bar{\nu}_{\mu} \mu^{-}\right) & \left(K^{-} \rightarrow \bar{\nu}_{\mu} \mu^{-}\right) & \left(K^{-} \rightarrow \pi^{0} \bar{\nu}_{\mu} \mu^{-}\right)
\end{array}
$$

The dominant process leading to $\nu_{e}\left(\bar{\nu}_{e}\right)$ in the beam are $K_{e 3}^{+}\left(K_{e 3}^{-}\right)$decays.

The E815 neutrino flux Monte Carlo is based on the DECAY TURTLE program[16] and on charged $\pi$ and $K$ production data from Atherton et al. [17] as parameterized for thick targets by Malensek[18]. The DECAY TURTLE Monte Carlo contains all the details of the beam apertures and the positions and dominant multipole fields of the magnetic elements. The primary beam position and angle come from an analysis of the beamline SWIC's and SEED's over the entire run. 
Flux Adjustment Procedure in Monte Carlo
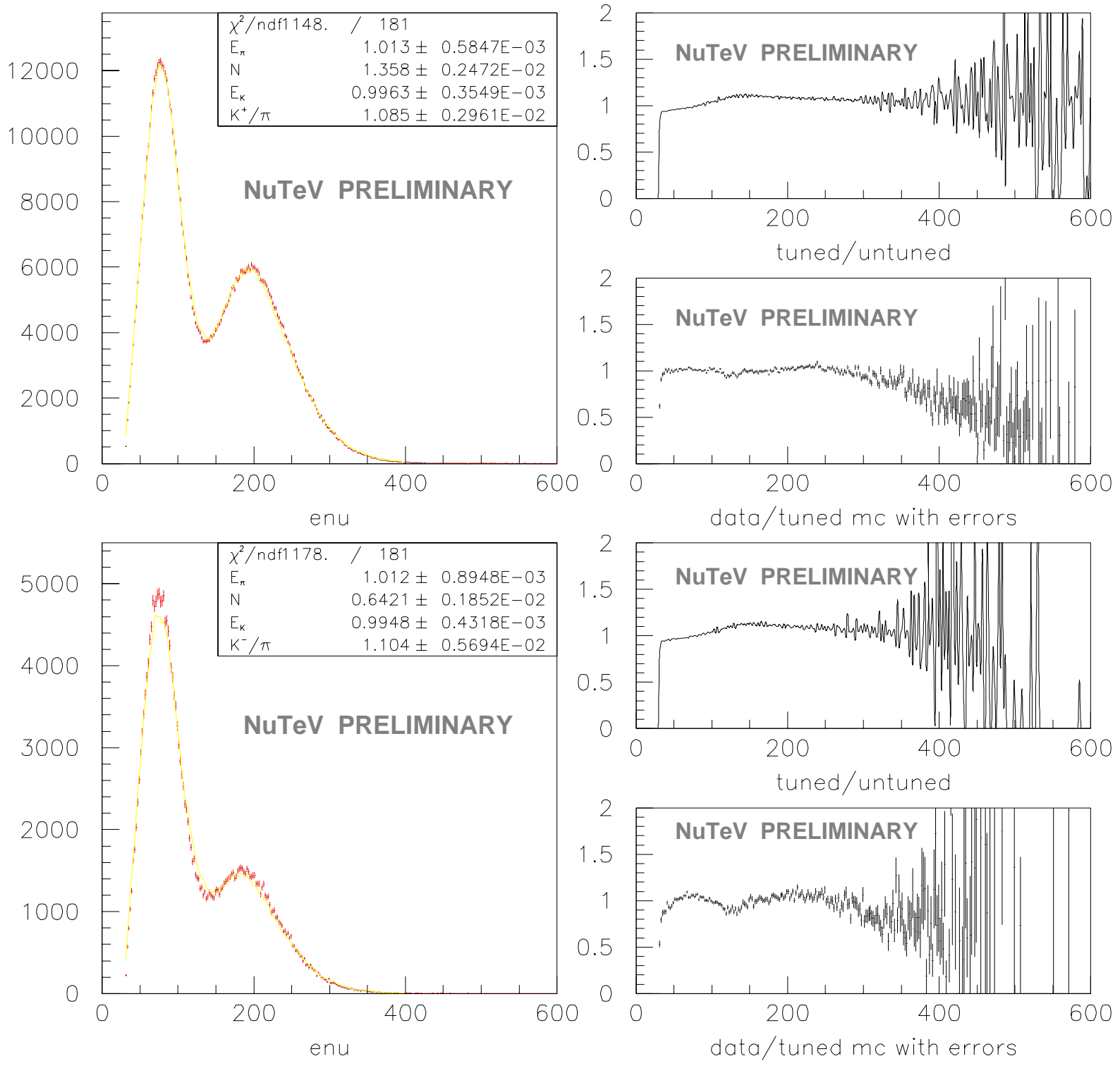

Figure 20: The flux tuning procedure for $E_{\nu}$. Shown is the fit for $K / \pi$ ratios and $\nu_{\pi}$ and $\nu_{K}$ energy scales and the residuals to the fits in both $\nu$ and $\bar{\nu}$ modes. The uncertainties in the plots are statistical uncertainties only. 


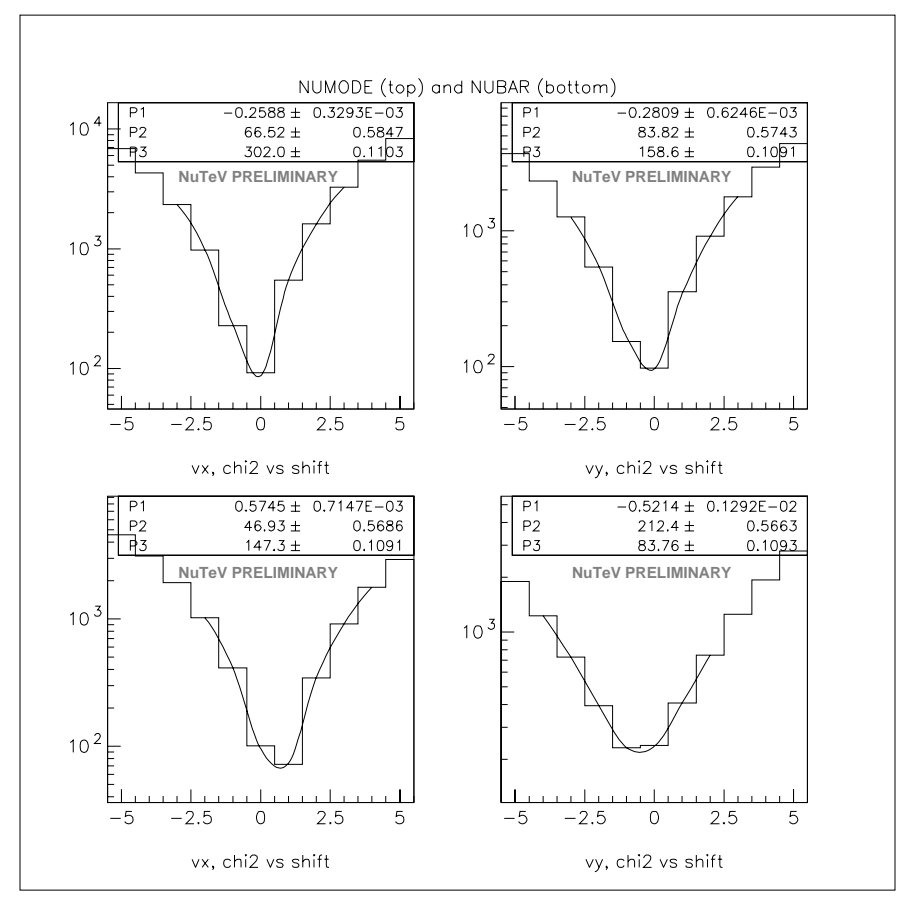

Figure 21: The flux tuning procedure for $\nu(\bar{\nu})$ beam position at the detector. Shown is the fit for $x$ and $y$ offsets in $\nu$ and $\bar{\nu}$ modes. The parameters $\mathrm{P} 1$, $\mathrm{P} 2$, and $\mathrm{P} 3$ are $\delta x$ or $\delta y$, the minimum $\chi^{2}$ value, and the second order term, respectively.

The flux tuning procedure for $\nu_{\mu}$ and $\bar{\nu}_{\mu}$ uses a broad sample of CC events. Figure 20 shows the observed $E_{\nu}$ of these events for neutrino mode (solid) and anti-neutrino (dashed) mode. The beam Monte Carlo $\nu_{\mu}$ and $\bar{\nu}_{\mu}$ fluxes from the three sources listed above are tuned by fitting the following parameters to the observed energy spectrum and neutrino interaction position in the data flux sample to absolute Monte Carlo predictions:

1. Monte Carlo beam position offsets in $x$ and $y$ views

2. The $K^{+} / \pi^{+}$and $K^{-} / \pi^{-}$production ratios

3. Energy scale factors $f_{E_{\nu}}^{X}$ where $X$ is $\pi^{+}, K^{+}, \pi^{-}, K^{-}$

The result of the tuning first for $E_{\nu}$ is shown in Figure 21, and the position tuning result is shown in Figure 22.

\subsection{Electron Neutrino Flux}

The electron neutrino flux prediction comes from applying the tuned parameters in the previous section to the beam Monte Carlo. The dominant source by far of $\nu_{e}$ and $\bar{\nu}_{e}$ in the beam is $K_{e 3}^{ \pm}$decay. The dominant uncertainty 
results from the poor knowledge of the $K^{ \pm} \rightarrow \pi^{0} e^{ \pm} \nu$ branching ratio, and data from $K^{+}$and $K_{L}$ decays are used to constrain it to a $1.1 \%$ uncertainty in Ref.[19].

$K_{L e 3}$ decays are also considered; these contribute at only about $10^{-2}$ of their charged counterparts because of the designed poor acceptance for neutrals in the SSQT[20].

Charmed mesons are also sources of $\nu_{e}$ which need to be accounted for in the flux simulation. They are produced in both the primary target and beam dump. Their short lifetime means they do not enter the SSQT, but still have some acceptance at the Lab E detector.

Production of charm mesons in both the proton dumps is a significant contribution to the total charm production by the primary beam. This has been modeled in the NuTeV beam Monte Carlo. The production weight for the primary beam on the beam dump has been calculated[21] and incorporated into the $\mathrm{NuTeV}$ Monte Carlo.

Electron neutrino production uncertainties are dominated by the branching ratio uncertainty of $1.1 \%$ for $K^{ \pm}$and by $20 \%$ error in the production cross section, for $K_{L}$ and charm. Since the level of $\nu_{e}$ contamination from $K_{L}$ and charm decays are two orders of magnitude lower than that from $K^{ \pm}$, this level of uncertainty from $\nu_{e}$ is sufficiently low for $\mathrm{NuTeV}$ to measure $\sin ^{2} \theta_{W}$ to an uncertainty equivalent to mass of the $W$ uncertainty of $100 \mathrm{MeV} / c^{2}$.

\subsection{Wrong Sign $\nu$ Contamination}

Since the SSQT provides clear distinctions in the running modes, it is relative straightforward to estimate what the fractional contamination of the wrong sign background is, in a given mode. Table 3 compares the measured number of wrong sign muon event fraction to the wrong sign fractions presented in TM-1884[3]. The cuts used to select good candidate events were energy cuts $\left(E_{\mu}>15 \mathrm{GeV}\right)$, detector fiducial volume cuts (event vertex within $\pm 50^{\prime \prime}$ from the center and muon within $64^{\prime \prime}$ radius at the front face of the toroid), and good muon quality cuts to ensure a good measurement of the muon momentum and charge.

Table 3: Measured wrong sign muon fractions for neutrino and anti-neutrino modes, compared to predictions of wrong sign neutrino fractions from the TM-1884[3].

\begin{tabular}{c|c|c} 
Running Mode & Measured fraction & Predicted fraction \\
\hline Neutrino Mode & $<1.08 \times 10^{-3}$ & $1.61 \times 10^{-3}$ \\
Anti-Neutrino Mode & $<2.9 \times 10^{-3}$ & $2.5 \times 10^{-3}$
\end{tabular}



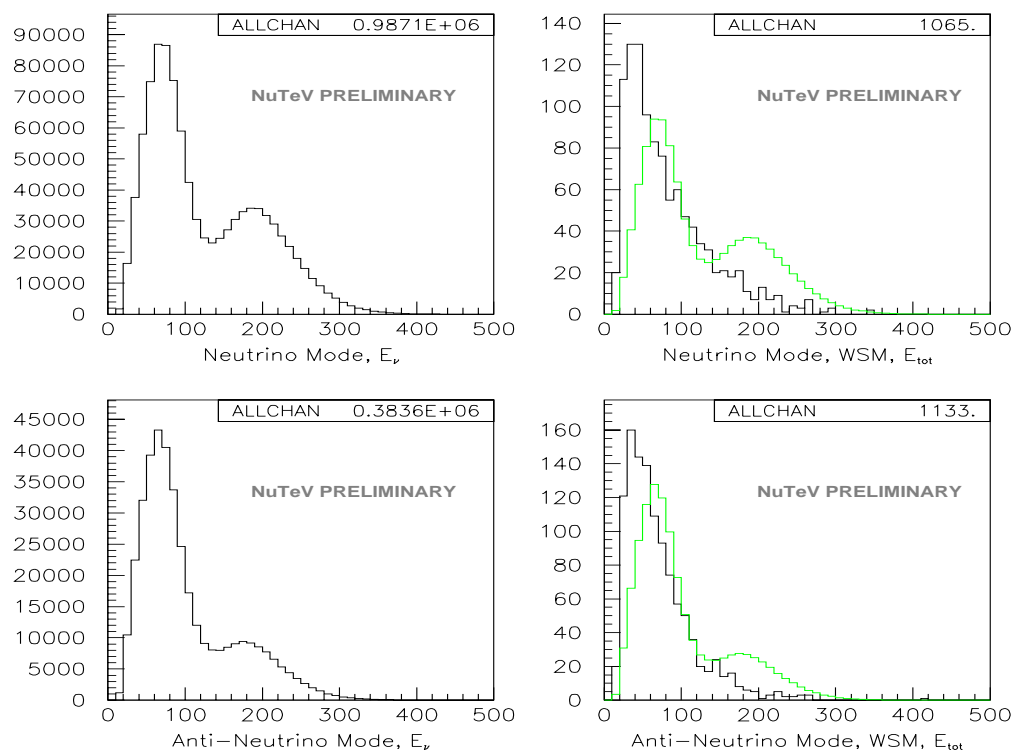

Figure 22: $E_{\nu}$ distributions of all (left) and wrong sign (right) candidates for neutrino (top) and anti-neutrino (bottom) modes. The dashed histograms in the wrong sign candidate histograms are the all candidate distributions normalized to the total number of wrong sign candidates for shape comparisons.
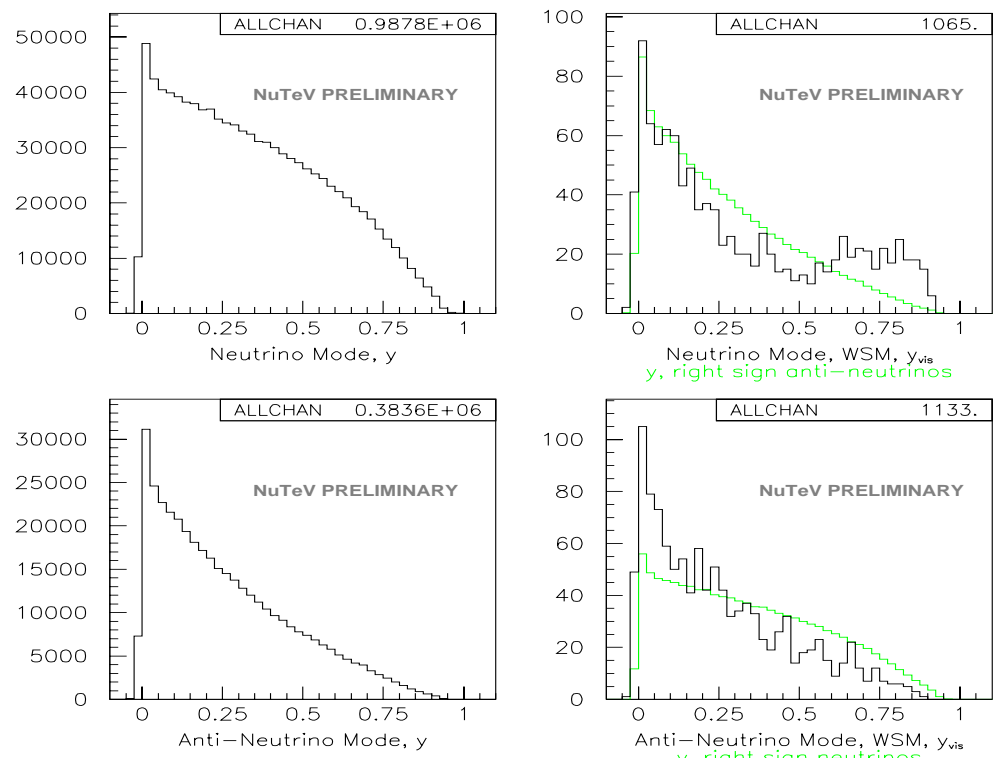

Figure 23: $y$ distributions of all (left) and wrong sign (right) candidates for neutrino (top) and anti-neutrino (bottom) modes. The dashed histograms in the wrong sign candidate histograms are the all candidate distributions normalized to the total number of wrong sign candidates for shape comparisons. 
Figure 23 shows neutrino energy $\left(E_{\nu}\right)$ distributions of all events passing the cuts and the wrong charge muon candidates for both neutrino (top) and anti-neutrino modes (bottom). The $E_{\nu}$ distributions display distinctively different characteristics for all candidates and the wrong sign muon candidates. Figure 24 shows the distributions of inelasticity $(y)$, defined as the fractional hadronic energy relative to $E_{\nu}$ between all candidates and wrong sign muon candidates. Here the wrong sign distributions are compared to the corresponding neutrino or anti-neutrino $y$ distributions to see if the distributions display expected characteristics of the wrong sign neutrinos resulting from the beam. The comparisons in $y$ distributions show that the the current wrong sign candidates are not all from the beam but rather a result of a mixture of various sources of wrong sign muons.

It should be noted that since the measured wrong sign muon fractions include other sources of wrong sign muons, such as charm production and $\pi$ and $K$ in-flight decays from hadronic showers resulting from an NC interactions, the fractions given in Table 3 are upper limits of the wrong sign neutrino background from the beamline. However, in both neutrino and anti-neutrino modes, the measured wrong sign muon fractions are consistent with or better than the predictions in TM-1884[3]. These fractions are lower than other experiments by approximately an order of magnitude and open up wide variety of physics possibilities, such as the investigation of quasi-elastic scattering and the charm sea. With the current understanding of the beam, $\mathrm{NuTeV}$ is now in a position to delineate the expected backgrounds and to start separating samples for physics measurements.

\section{Conclusions}

The E815 neutrino beamline has performed beyond the expectations and the monitors provided invaluable information in understanding the beam. The total number of protons delivered on target while DAQ running was $3.01 \times 10^{18}$, and the overall data taking efficiency was $95 \%$ without excluding the dead time due to veto, resulting in $2.86 \times 10^{18}$ PoT recorded on tape.

The upper limit levels of wrong sign background are in good agreement with the initial expectation of TM-1884. The $\nu_{e}$ backgrounds have been constrained to acceptable levels for the $\sin ^{2} \theta_{W}$ analysis. The SSQT in general has performed up to and beyond its expectation and the experiment is well on its way to producing results from the data.

\section{Acknowledgements}

We would like to express our gratitude to the US Department of Energy for supporting the construction and maintenance of the beamline over the 
past fixed target run. We express our deep appreciation to Fermilab for providing all the necessary technical support in this experiment. We thank the numerous people who helped in all the facets of this project: the Beams Division, the engineering, mechanical, electrical and survey and alignment groups.

\section{References}

[1] C.H.Llewellyn Smith, Nucl. Phys. B228, 205 (1983)

[2] Bruce King, Ph.D. Thesis, Columbia University (1993).

[3] R.H.Bernstein, NuTeV Collaboration, "Technical Memorandum: Sign Selected Quadrupole Train", FERMILAB-TM-1884 (1994).

[4] E.A.Paschos and L. Wolfenstein, Phys. Rev. D7, 91 (1973)

[5] W. Seligman, "A Next-to-Leading-Order QCD Analysis of Neutrino-Iron Structure Functions at the Tevatron," Ph.D. Thesis, Columbia University, unpublished (1997)

[6] K.S.McFarland and R.B.Drucker, NuTeV internal note, "SSQT alignment requirements" (1994).

[7] K.S.McFarland, R.B.Drucker, D.A.Harris, and J. Yu, NuTeV internal note, "Run Plan for the NuTeV SSQT 150 GeV Alignment Run" (1996).

[8] Fermilab Beam's Division Operations Web page at the URL : "http://www-bd.fnal.gov/operations/fxtar/qxr_fast.html" (1997)

[9] R.B.Drucker, G. Tassotto, R.Ford et. al., FERMILAB-TM-2024, in preparation.

[10] J. Krider \& C. Hojvat, "A Multiwire Secondary Emission Beam Profile Monitor with $20 \mu \mathrm{m}$ resolution," Nucl. Inst. and Meth. A247, 304-308 (1986).

[11] J. Camas, G. Ferioli, J.J. Gras, R. Jung, "Screen Versus Sem Grids for Single Pass Measurements in SPS, LEP and LHC," DESY M-9507, June (1995).

[12] G. Tassotto, "Beam Profile Monitors for High Intensity Proton Beams", AIP Conference Proceedings 309, Argonne IL, May 6-9, 1996, 506 (1996).

[13] R.B.Drucker, "BPM performances in the NuTeV," NuTeV Internal note, in preparation (1998). 
[14] R.B.Drucker and M.Vakili, Fermilab-TM-2025, in preparation.

[15] W. Lefman, K. McFarland, A. Romosan, "Removing Muon-Induced Events in the Neutral Current Analysis", NuTeV Internal Note, April (1995).

[16] SLAC-246, March 1982.

[17] Atherton et al, CERN 80-70.

[18] A.J. Malensek, "Empirical Formula for Thick Target Particle Production", FN-341 (1981).

[19] K. McFarland, "Electron Neutrinos from Charged Kaons", NuTeV Internal Note, September (1994).

[20] R. Bernstein, T. Bolton, J. Conrad, R. Johnson and M. Vakili, "Electron Neutrinos from $K_{L} \mathrm{~s}$ ", NuTeV Internal Note, April (1994).

[21] T.Bolton, "Some Analytic $\nu_{e}$ Flux Calculations", NuTeV Internal note, (1998). 